ACCEPTED MANUSCRIPT

\title{
Plasma-sheath transition in multi-fluid models with inertial terms under low pressure conditions: Comparison with the classical and kinetic theory
}

To cite this article before publication: Alejandro Alvarez-Laguna et al 2019 Plasma Sources Sci. Technol. in press https://doi.org/10.1088/1361$\underline{6595 / a b 6242}$

\author{
Manuscript version: Accepted Manuscript \\ Accepted Manuscript is "the version of the article accepted for publication including all changes made as a result of the peer review process, \\ and which may also include the addition to the article by IOP Publishing of a header, an article ID, a cover sheet and/or an 'Accepted \\ Manuscript' watermark, but excluding any other editing, typesetting or other changes made by IOP Publishing and/or its licensors"
}

This Accepted Manuscript is @ 2019 IOP Publishing Ltd

During the embargo period (the 12 month period from the publication of the Version of Record of this article), the Accepted Manuscript is fully protected by copyright and cannot be reused or reposted elsewhere.

As the Version of Record of this article is going to be / has been published on a subscription basis, this Accepted Manuscript is available for reuse under a CC BY-NC-ND 3.0 licence after the 12 month embargo period.

After the embargo period, everyone is permitted to use copy and redistribute this article for non-commercial purposes only, provided that they adhere to all the terms of the licence https://creativecommons.org/licences/by-nc-nd/3.0

Although reasonable endeavours have been taken to obtain all necessary permissions from third parties to include their copyrighted content within this article, their full citation and copyright line may not be present in this Accepted Manuscript version. Before using any content from this article, please refer to the Version of Record on IOPscience once published for full citation and copyright details, as permissions will likely be required. All third party content is fully copyright protected, unless specifically stated otherwise in the figure caption in the Version of Record.

View the article online for updates and enhancements. 


\title{
Plasma-sheath transition in multi-fluid models with inertial terms under low pressure conditions: Comparison with the classical and kinetic theory
}

\author{
Alejandro Alvarez Laguna ${ }^{1,2}$, Thierry Magin ${ }^{1,3}$, Marc Massot ${ }^{2}$, \\ Anne Bourdon ${ }^{1}$, and Pascal Chabert ${ }^{1}$ \\ ${ }^{1}$ Laboratoire de Physique des plasmas, CNRS, Ecole Polytechnique, Sorbonne \\ Université, Univ. Paris Sud, 91128, Palaiseau, France \\ ${ }^{2}$ Centre de Mathématiques Appliquées, CNRS, Ecole Polytechnique, Institut \\ Polytechnique de Paris, 91128 Palaiseau, France. \\ ${ }^{3}$ von Karman Institute for Fluid Dynamics, Chaussée de Waterloo, 72, B-1640 \\ Rhode-St-Gense, Belgium \\ E-mail: alejandro.alvarez-laguna@polytechnique.edu
}

\begin{abstract}
We present high-order accuracy simulations of the plasma-sheath transition with an ion-electron multi-fluid model that considers the electron inertial terms and the ion pressure gradient. By means of a third-order accuracy timedependent finite volume scheme, we solve the isothermal multi-fluid equations with realistic ion-to-electron mass ratios. We propose a numerical procedure and boundary conditions that retrieve a steady-state solution of a planar 1D floating sheath. The classical solution to this problem neglects the ion temperature since the model equations present a singularity for a finite ion temperature. The multi-fluid simulations provide a rigorous solution to the plasma-sheath transition without singularities. We compare our solution to the classical theory, finding perfect agreement when the ionto-electron temperature tends to zero. We discuss the effect of the ion temperature, the electron inertia, and the elastic collisions with neutrals in low-pressure plasmas. Finally, relying on a kinetic approach, we derive analytical expressions for the electron macroscopic quantities inside a steady sheath that assumes a truncated Maxwellian electron velocity distribution function (EVDF) with a wall that collects the electron random flux. The derived analytical expressions are beyond the classical isothermal solution with Boltzmann electrons. The isothermal multi-fluid solution captures properly the first two moments of the EVDF inside the sheath. Our analytical expressions show the need for solving for higher-order moments to fully explain the electron physics inside the sheath, as opposite to the classical isothermal assumption. This work demonstrates that the multi-fluid simulations are able to capture the plasmasheath transition under weakly collisional conditions with a solution that is consistent with the classical and the kinetic theory.
\end{abstract}

Keywords: Sheath; Multi-Fluid; High-order methods; Discharge; Floating Sheath; 


\section{Introduction}

The study of the plasma-sheath transition is fundamental to a wide number of technological applications: magnetically confined fusion [1], plasma processing [2], plasma diagnostics [3], plasma arcs [4] or electric propulsion [5]. The transition between the sheath and the bulk of the plasma in a simple plasma composed by positiveions and electrons is a long-standing problem $[6,7,8]$ that has been studied through analytical models [8, 9], Particle-in-cell (PIC) simulations [10], kinetic simulations [11], fluid models, [12], and hybrid models [13]. An extensive review on the different models and resolution methods of a plasma sheath in laboratory and space plasmas is given by Robertson [14]. However, the self-consistent simulations of sheaths in fluid numerical simulations of low-pressure plasmas is still nowadays an open question due to the kinetic processes that occur in the sheath and the boundary conditions used in the computational model $[15,16]$.

The present paper is aimed at the simulation of sheaths with fluid models under lowtemperature and low-pressure conditions. In particular, we consider fluid models that do not neglect electron inertia nor the ion temperature. These models are commonly referred to as multi-fluid. The electron inertia is no longer negligible when the electrons are accelerated to high speeds that are comparable to the electron thermal speeds. In that case, the electron fluid cannot be modelled with a Boltzmann relation. Recent numerical and experimental works have reported electron inertial effects in electron sheaths and presheaths [17, 18, 19], plasma sheath instabilities [20], Langmuir probes [21], magnetized plasmas [22, 23, 24], and Hall thrusters instabilities [25]. Similarly, the effect of the ion temperature on the plasma-wall interaction has been studied experimentally [26] and analytically [27], showing small deviations from the classical theory as the ion-to-electron temperature ratio increases.

Numerical simulations that are based on the fluid description, while including the electron inertia, are rather unexplored in low-pressure plasma discharges. Previous works [28, 29, 30, 31] have proposed numerical schemes for the multi-fluid equations coupled to Maxwell's equations for different applications, ranging from nuclear fusion and low-temperature discharges to astrophysical scenarios. However, most of these works do not include the transition between the plasma and the sheath in bounded plasmas. A recent paper by Cagas et al. [12] shows that multi-fluid simulations are able to reproduce the physics of collisionless plasma sheaths, with comparable results to continuum kinetic simulations. Nevertheless, in this study, with a time-evolving solver, the authors obtain a quasi-steady state solution for a hydrogen plasma that has an electron-to-ion mass ratio much smaller than typical low-temperature discharges. Furthermore, the comparison between the multi-fluid and the classical theory solution has not been provided in the literature heretofore. For this reason, we believe that the comparison with the classical theory as well as the study with heavier ions can be considered as an important first step towards realistic dynamic multi-fluid simulations of bounded plasmas. 
In this paper, we study the plasma-sheath transition of a planar 1D floating sheath with a multi-fluid model. This work is targeted towards three main goals. First, we aim at benchmarking the numerical method and boundary conditions for the multifluid equations in the simulation of low-pressure bounded plasmas composed by heavy species. Multi-fluid models can be an alternative to the drift-diffusion approximation that fails to represent weakly-collisional plasmas, especially when the electrons have a large drift velocity caused, for instance, by a magnetic field. Similarly, the solution of higher-order moments of the Boltzmann equation can capture kinetic effects, being a sound alternative that might be cheaper than kinetic simulations. Second, we compare the solution with the analytical classical collisionless theory. The classical collisionless theory [8] considers Boltzmann electrons and mono-energetic (pressureless) ions. This classical theory has two main shortcomings: (1) the analytical/solutions of the sheath and the presheath do not join smoothly due to a singularity [8] and (2) the equations are singular in the presheath when the positive-ion temperature is considered [27]. In our paper, we prove that these singularities are not present when the complete timedependent system of partial differential isothermal equations is solved to steady-state. This provides a rigorous solution of the classical plasma-wall transition without the need of matching [8] or patching both solutions [32, 33], nor asymptotic expansions [27].

Finally, the third goal of this paper is to link the multi-fluid solution of a collisionless plasma sheath with the kinetic theory. The analytical expression of the electron velocity distribution function (EVDF) in a collisionless sheath ean be obtained with the theory of characteristics $[34,10]$. By assuming that the wall collects the random flux of electrons, the resulting EVDF is a velocity-space truncated distribution. In this work, we show the consequences of the truncation of the distribution function on the different fluid moments, providing analytical expressions for the fluid quantities, and we compare them to the multi-fluid solution obtained by our non-linear solver.

\section{Model equations}

We consider a plane, symmetric discharge that contains a constant neutral gas background and two charged species that are treated as fluids: cold positive ions at a temperature $T_{\mathfrak{i}}$ and electrons with a temperature $T_{\mathfrak{e}}$. As in the classical theory, the species are considered to be at constant temperature that is homogeneous in space. The computational domain is one-dimensional and extends from $x=-L / 2$ to $x=L / 2$. The charged species are created by ionization and two-floating walls are located at $x= \pm L / 2$.

The system of equations in dimensional form reads as follows

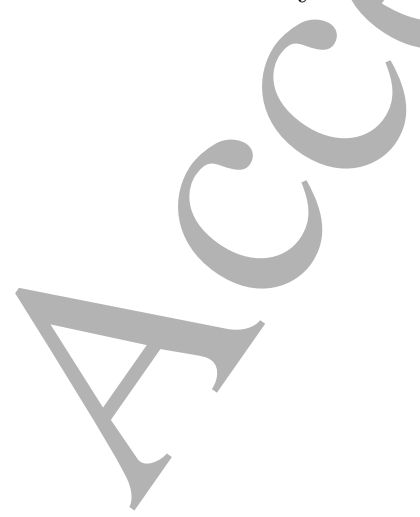




$$
\begin{aligned}
& \frac{\partial}{\partial t} n_{\mathfrak{e}}+\frac{\partial}{\partial x}\left(n_{\mathfrak{e}} u_{\mathfrak{e}}\right)=n_{\mathfrak{e}} \nu^{i z} \\
& \frac{\partial}{\partial t} n_{\mathfrak{i}}+\frac{\partial}{\partial x}\left(n_{\mathfrak{i}} u_{\mathfrak{i}}\right)=n_{\mathfrak{e}} \nu^{i z}, \\
& m_{\mathfrak{e}} \frac{\partial}{\partial t}\left(n_{\mathfrak{e}} u_{\mathfrak{e}}\right)+\frac{\partial}{\partial x}\left(m_{\mathfrak{e}} n_{\mathfrak{e}} u_{\mathfrak{e}}^{2}+p_{\mathfrak{e}}\right)=n_{\mathfrak{e}} e \frac{\partial \phi}{\partial x}-m_{\mathfrak{e}} u_{\mathfrak{e}} n_{\mathfrak{e}} \nu_{\mathfrak{e n}}, \\
& m_{\mathfrak{i}} \frac{\partial}{\partial t}\left(n_{\mathfrak{i}} u_{\mathfrak{i}}\right)+\frac{\partial}{\partial x}\left(m_{\mathfrak{i}} n_{\mathfrak{i}} u_{\mathfrak{i}}^{2}+p_{\mathfrak{i}}\right)=-n_{\mathfrak{i}} e \frac{\partial \phi}{\partial x}-m_{\mathfrak{i}} u_{\mathfrak{i}} n_{\mathfrak{i}} \nu_{\mathfrak{i n}}, \\
& \frac{\partial^{2} \phi}{\partial x^{2}}=\frac{n_{\mathfrak{e}}-n_{\mathfrak{i}}}{\epsilon_{0}} e
\end{aligned}
$$

where $n_{\mathfrak{e}}$ and $n_{\mathfrak{i}}$ stand for the electron and ion number density respectively, $u_{\mathfrak{e}}$ and $u_{\mathfrak{i}}$ the electron and ion velocities, and $\phi$ the electric potential. The ionization frequency is denoted as $\nu^{i z}$ whereas the ion-neutral and electron-neutral elastie collision frequency are $\nu_{\mathfrak{e n}}$ and $\nu_{\mathfrak{i n}}$, respectively. The model neglects recombination and Coulomb collisions. The pressures of the electron and ion fluids are assumed to obey the perfect gas law, $p_{\mathfrak{e}}=n_{\mathfrak{e}} \mathrm{k}_{\mathrm{B}} T_{\mathfrak{e}}$ and $p_{\mathfrak{i}}=n_{\mathfrak{i}} \mathrm{k}_{\mathrm{B}} T_{\mathfrak{i}}$, where $\mathrm{k}_{\mathrm{B}}$ is Boltzmann's constant.

To normalize the model, we introduce the following reference quantities:

$$
\begin{aligned}
& \bar{t}=t / t_{0}, \bar{x}=x / L_{0}, \\
& \bar{n}_{\mathfrak{i}}=n_{\mathfrak{i}} / n_{0}, \bar{n}_{\mathfrak{e}}=n_{\mathfrak{e}} / n_{0}, \bar{u}_{\mathfrak{e}}=u_{\mathfrak{e}} / u_{0}, \bar{u}_{\mathfrak{i}}=u_{\mathfrak{i}} / u_{0}, \bar{\phi}=\phi / \phi_{0}, \\
& \bar{\nu}^{i z}=t_{0} \nu^{i z}, \bar{\nu}_{\mathfrak{e n}}=\nu_{\mathfrak{e n}} t_{0}, \bar{\nu}_{\mathfrak{i n}}=\nu_{\mathfrak{i n}} t_{0},
\end{aligned}
$$

where $n_{0}$ is the initial ion number density, $L_{0}=L$ is the distance between plates, $u_{0} \equiv u_{B}=\sqrt{\mathrm{k}_{\mathrm{B}} T_{\mathfrak{e}} / m_{\mathfrak{i}}}$ is the classical Bohm velocity, the charateristic time $t_{0}=L_{0} / u_{0}$ is obtained from the reference velocity and reference distance, $\lambda_{D 0}=\left(\epsilon_{0} \mathrm{k}_{\mathrm{B}} T_{\mathfrak{e}} / e^{2} n_{0}\right)^{1 / 2}$ is the Debye length computed with the initial density, and the reference potential $\phi_{0}=\mathrm{k}_{\mathrm{B}} T_{\mathfrak{e}} / e$ is the electron temperature in $\mathrm{eV}$. The normalized set of equations reads

$$
\begin{aligned}
& \frac{\partial}{\partial \bar{t}} \bar{n}_{\mathfrak{e}}+\frac{\partial}{\partial \bar{x}}\left(\bar{n}_{\mathfrak{e}} \bar{u}_{\mathfrak{e}}\right)=\bar{n}_{\mathfrak{e}} \bar{\nu}^{i z}, \\
& \frac{\partial}{\partial \bar{t}} \bar{n}_{\mathfrak{i}}+\frac{\partial}{\partial \overline{\bar{x}}}\left(\bar{n}_{\mathfrak{i}} \bar{u}_{\mathfrak{i}}\right)=\bar{n}_{\mathfrak{e}} \bar{\nu}^{i z} \\
& \frac{\partial}{\partial \bar{t}}\left(\bar{n}_{\mathfrak{e}} \bar{u}_{\mathfrak{e}}\right)+\frac{\partial}{\partial \bar{x}}\left[\bar{n}_{\mathfrak{e}}\left(\bar{u}_{\mathfrak{e}}^{2}+\varepsilon^{-1}\right)\right]=\frac{\bar{n}_{\mathfrak{e}}}{\varepsilon} \frac{\partial \bar{\phi}}{\partial \bar{x}}-\bar{\nu}_{\mathfrak{e n}} \bar{n}_{\mathfrak{e}} \bar{u}_{\mathfrak{e}} \\
& \frac{\partial}{\partial \bar{t}}\left(\bar{n}_{\mathfrak{i}} \bar{u}_{i}\right)+\frac{\partial}{\partial \bar{x}}\left[\bar{n}_{\mathfrak{i}}\left(\bar{u}_{\mathfrak{i}}^{2}+\kappa\right)\right]=-\bar{n}_{\mathfrak{i}} \frac{\partial \bar{\phi}}{\partial \bar{x}}-\bar{\nu}_{\mathfrak{i n}} \bar{n}_{\mathfrak{i}} \bar{u}_{\mathfrak{i}} \\
& \frac{\partial^{2} \bar{\phi}}{\partial \bar{x}^{2}}=\frac{\bar{n}_{\mathfrak{e}}-\bar{n}_{\mathfrak{i}}}{\lambda^{2}},
\end{aligned}
$$

where the electron-ion mass ratio is introduced as $\varepsilon=m_{\mathfrak{e}} / m_{\mathfrak{i}}$, the ion-to-electron temperature ratio is $\kappa=T_{\mathfrak{i}} / T_{\mathfrak{e}}$, and the normalized initial Debye length is $\lambda=\lambda_{D 0} / L_{0}$.

The proposed system of equations needs to be solved as an initial-boundary-value problem and therefore both initial and boundary conditions need to be specified. To obtain the stationary solution for the plasma-sheath transition, the time-dependent 
equations are evolved to steady state. A static electrically-neutral homogeneous profile is used as initial condition, as follows,

$$
\left.\left(\bar{n}_{\mathfrak{e}}, \bar{n}_{\mathfrak{e}} \bar{u}_{\mathfrak{e}}, \bar{n}_{\mathfrak{i}}, \bar{n}_{\mathfrak{i}} \bar{u}_{\mathfrak{i}}, \bar{\phi}\right)\right|_{t=0}=\left(\begin{array}{ll}
1,0,1,0,0
\end{array}\right) .
$$

Concerning the boundary conditions, in order to have a well-posed problem, not all the combinations of boundary conditions for the different variables lead to a consistent solution (see, e.g., $[35,36])$. In the case of a floating plasma sheath, the electrons flow at speeds lower than their thermal velocity at the boundary, i.e., at subsonic speeds. Therefore, one of the characteristics of the associated Riemann problem will propagate from the boundary inwards the domain. For this reason, we can only specify one of the variables at the boundary. Alternatively, the ions flow at speeds larger than Bohm's speed (i.e., supersonic) and, therefore, all the information of the ion fluid propagates outwards the domain. This means that none of the ion variables can be consistently specified at the boundaries. Consequently, we specify that the flux of electrons leaving the domain at the wall is equal to the random flux, which in physical and normalized units reads,

$$
\left.n_{\mathfrak{e}} u_{\mathfrak{e}}\right|_{\text {wall }}=\left.n_{\mathfrak{e}}\right|_{\text {wall }} \sqrt{\frac{\mathrm{k}_{\mathrm{B}} T_{\mathfrak{e}}}{2 \pi m_{\mathfrak{e}}}} \quad \text { and }\left.\quad \bar{n}_{\mathfrak{e}} \bar{u}_{\mathfrak{e}}\right|_{\text {wall }}=\left.\bar{n}_{\mathfrak{e}}\right|_{\text {wall }} \sqrt{\frac{1}{2 \pi \varepsilon}} .
$$

This flux assumes that the distribution function of the electrons at the edge of the sheath is a Maxwellian and the wall absorbs all the incoming electrons. No secondary electron emission is considered in this model. For the ion fluid, as they flow at velocities faster than Bohm's speed at the wall, an "outflow boundary" (Neumann condition) is used. Finally, we impose $\bar{\phi}_{\text {wall }}=0$ for the potential. The implementation of the boundary conditions into the numerical solver through the ghost cell strategy is explained in Appendix A.

As in the classical theory, the ionization frequency is an eigenvalue of the problem [8] in order to obtain a steady-state solution. This eigenvalue problem is a result of the balance between the production of ions in the bulk of the plasma and the losses to the walls, coupling the physics of the sheath and the pre-sheath. In our numerical set-up, the eigenvalue is found by fixing an ionization frequency that balances the flux of ions through the wall at every time step. Consequently, the ionization frequency is imposed at the time step $\bar{t}^{n}$ as

$$
\bar{\nu}^{i z}\left(\bar{t}^{n}\right)=\frac{\left.\left(\bar{n}_{\mathfrak{i}} \bar{u}_{\mathfrak{i}}\right)\right|_{\bar{x}=1 / 2}-\left.\left(\bar{n}_{\mathfrak{i}} \bar{u}_{\mathfrak{i}}\right)\right|_{\bar{x}=-1 / 2}}{\int_{-1 / 2}^{1 / 2} \bar{n}_{e}\left(\bar{t}^{n}\right) d \bar{x}} .
$$

By doing this, the average density of the ions remains to unity during the full simulation as the losses at the wall are compensated by the ionization.

\subsection{Differences with the classical fluid theory of floating sheath}

The classical fluid model of floating sheath and its resolution method is outlined by Riemann [8] and Robertson [14]. The system of fluid equations that we solve in this 
paper has fundamental differences compared to these references, that we would like to highlight in this section. These differences are summarised as follows:

(i) The classical fluid model for the sheath solves for the steady-state system of equations, i.e., the time-dependent part of the simplified fluid equations is not taken into account. This leads to a system of ordinary differential equations (ODEs) that is solved as an initial-value problem. This resolution method is very appropriate to obtain an approximation of the sheath solution but has strong limitations, as it will be explained below. Here, we consider a system of partial differential equations (PDEs) that contain the time-dependent part and is solved as an initial-boundaryvalue problem. The system is converged to steady state but, as the reader may notice, the numerical method is also valid to study the evolution of ion-electron systems in bounded domains.

(ii) The steady-state system of ODEs of the classical theory presents some limitations in order to be resolved, as explained in both references [8, 14]. First, both methods present a singularity at the center (caused by $u_{\mathfrak{i}}=0$ ) and, moreover, the system of equations is singular for finite ion temperature (as explained in, e.g., [27]). Second, the size of the domain cannot be specified in the simulation as the ionization source and the length of the domain cannot be chosen independently. For this reason, in the case of Robertson, the value of the floating potential needs to be chosen, and in the case of Riemann [8], the domain is ended at the point where the ions reach the wall flux (usually this value is the random flux of electrons). Due to these limitations, they cannot be extended to multiple dimensions nor to be used in unsteady problems. This can be regarded as a fundamental difference to our method that does not contain singularities and can be applied to unsteady problems, being easily extended to/multi-dimensions. Moreover, the floating potential is not imposed as a boundary condition and is found self-consistently in the simulations.

(iii) The system of fluid equations of the classical theory contains assumptions that are not made in the present paper: the ions are considered as mono-energetic, and the electrons follow a Boltzmann distribution. Additionally, the method shown by Robertson [14] considers the ionization source as homogeneous in space. On the other hand, the equations we solve in the paper account for the ion temperature, the electron inertia, and the ionization source is not homogeneous in space as it depend on the electron density.

However, the fact of considering a more general system of equations results in a physical model that inherently contains a wide span of time and space scales and, thus, the system becomes stiff and difficult to be solved numerically. Nevertheless, the proposed methodology is more general than the classical fluid sheath and therefore can be-extended to unsteady problems, to multi-dimensions, and higher-order moment system of equations. 


\section{Numerical method and simulation set-up}

We propose to solve the system of equations (7)-(11) by means of an unsteady finite volume method for the fluid equations and a finite difference method for the Poisson equation. The proposed numerical scheme is implemented in a single dimension and uses the Roe scheme [37] for the convective fluxes with third-order accuracy total variation diminishing (TVD) reconstruction $[38,39]$ that is using the flux limiter proposed by [40]. Thomas's algorithm is used to solve Poisson's equation, and a third-order accuracy TVD Runge Kutta schemes for the time integration [41]. In order to impose the boundary conditions, as explained in the previous section, in the finite volume method, we use the ghost cell approach. A detailed description of the numerical procedure is given in Appendix A.

In order to guarantee numerical stability, the time step of the simulation needs to resolve the electron scales $[42,28]$, which means that both the electron sound waves and the plasma waves need to be resolved, i.e.,

$$
\begin{aligned}
& \Delta t \leq \min \left(\Delta x / \bar{u}_{c_{\max }}^{\mathfrak{e}}, \omega_{p e}^{-1}\right) \\
& \quad \text { with }\left|\bar{u}_{c_{\max }}^{\mathfrak{e}}\right|=\max \left(\left|\bar{u}_{e}+\varepsilon^{-1 / 2}\right|,\left|\bar{u}_{e}-\varepsilon^{-1 / 2}\right|\right) \text { and } \omega_{p e}=\left(\lambda^{2} \varepsilon / \bar{n}_{\mathfrak{e}}\right)^{-1 / 2} .
\end{aligned}
$$

The time-step in this paper is chosen to be $\Delta t=0.9 \min \left(\Delta x / \bar{u}_{c_{\max }}^{\mathfrak{e}}, \omega_{p e}^{-1}\right)$.

From the previous stability condition, one can notice that multi-fluid solvers are a multi-scale problem with numerical constraints that are similar to those of PIC codes. As discussed in [31], the numerical difficulties become more challenging for large ionto-electron mass ratios and small Debye lengths. Our numerical experiments show the importance of using high-order schemes for both the time and space discretization in order to avoid erroneous solutions for the electron flux. High-order TVD schemes have low numerical dissipation while avoiding spurious numerical oscillations, which is seen to be fundamental in low-pressure multi-fluid codes. Similarly, the mesh needs to properly resolve the Debye length in order to capture the sheath, even in high-order accuracy. In our paper we use a resolution of $\Delta x=0.1 \lambda_{D 0}$.

We decide to start the simulation from a flat density profile that does not assume any solution. This choice is oriented to prove the robustness and stability of the methodology to find the sheath solution. Note that an ill-conditioned numerical scheme would diverge for initial solutions that are not close enough to the final solution. In the simulations, the transient until convergence evolves as explained below. In the first time steps, the initial homogenous quasi-neutral plasma without electric field is modified close to the boundaries as a result of the random flux of electrons through the walls. The loss of electrons produces a charge separation in the vicinity of the boundaries that creates a positive potential that pushes the ions towards the walls. In this narrow region, the potential grows self-consistently until the ion flux equals the electron flux at the surface, so no dc current is drawn from the plasma. The perturbation of the initial field produces electron plasma waves that propagate towards the center. These waves are eventually convected outside the domain after $0.3-0.5$ Bohm speed crossing 

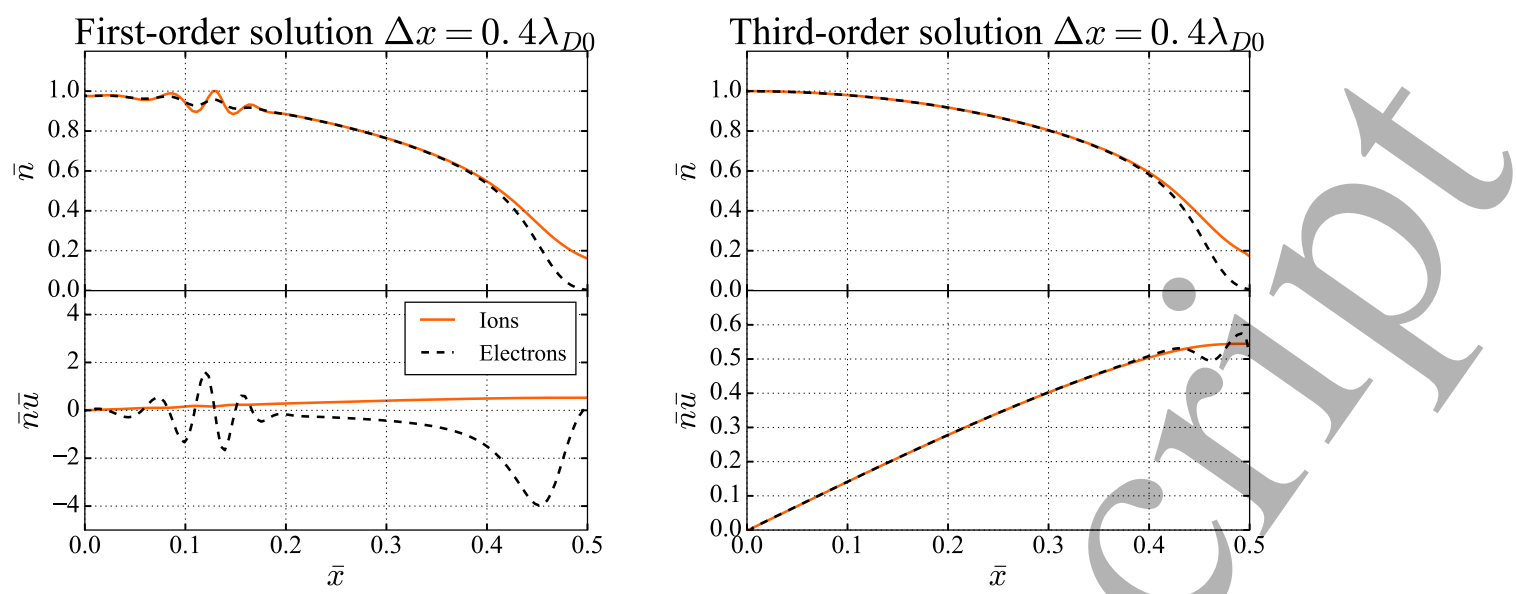

Figure 1. Example of erroneous solutions of the multi-fluid ion-electron plasmasheath transition that are result of a poor spatial resolution or a poor numerical scheme accuracy. The simulations consider $m_{\mathfrak{e}} / m_{i}=1.37 \cdot 10^{-5}, T_{\mathfrak{i}} / T_{\mathfrak{e}}=0.025, L=100 \lambda_{D 0}$, and $\bar{\nu}_{\mathfrak{e n}}=\bar{\nu}_{\mathfrak{i n}}=0$. Left panel: normalized densities (top) and fluxes (bottom) at $t=150 \omega_{p e_{0}}^{-1}$ obtained by a first-order accuracy solution in space and time. Right: converged solution with third-order accuracy in space and time solver using a poor spatial resolution.

times, i.e., $\bar{t}=0.3-0.5$. The solution remains quasi-neutral in the bulk of the domain, where the ionization evolves to compensate the losses at the walls. The full simulation converges after approximately $\bar{t}=3-4$. We set the convergence criterion when the residual of the unsteady part is smaller than $10^{-4}$. This corresponds to $10^{6}$ to $10^{7}$ iterations, depending of the size of the domain and the ion-to-electron mass ratio.

An example of the importance of high-order accurate schemes and the spacial resolution is illustrated in Fig. 1. In this figure, we present a first-order and third-order solutions accurate with $\Delta x=0.4 \lambda_{D 0}$ (i.e., 2.5 points per initial Debye length). The chosen conditions correspond to a typical Argon discharge, i.e., $\varepsilon=1.37 \cdot 10^{-5}, \kappa=0.025$, and $L=100 \lambda_{D 0}$. In Fig. 1, we present both the density and the fluxes of ions and electrons. The first-order solution (left panel of Fig. 1) is obtained with $\Delta t=0.01 \omega_{p e}^{-1}$. We observe that it develops spurious instabilities at the position where the ions cross the ion thermal velocity. These instabilities oscillate in time and grow until saturation. Moreover, the electron flux is not equal to the ion flux due to the large numerical dissipation of the first order scheme. Alternatively, the third-order solution (right panel of Fig. 1) has no oscillations and the electron flux equals the ion flux in the quasi-neutral region. However, the electron flux is not properly captured in the sheath due to the poor resolution. As a result, these numerical problems illustrate the difficulties of solving the electron moment equations with large ion-to-electron mass ratios. These problems are resolved by using highly-resolved high order accuracy discretizations, as it will be shown in the following.

In this paper, we propose a third-order spatial scheme for two main reasons. First, the use of higher-order methods in finite volume schemes is usually limited by the size 
of the stencil used for the reconstruction. As we are interested in the sheath solution, the high-order reconstruction becomes problematic at the boundary of the domain, as there are not enough elements to perform the reconstruction. For this reason, second order or the proposed third order is beneficial as it only uses a one-cell stencil to do the reconstruction. Therefore, at the boundary we can perform the reconstruction with only one ghost cell.

A less obvious advantage of the third-order accuracy is that odd-order $(1,3,5 \ldots)$ accuracy schemes are less dispersive than even-order $(2,4,6 \ldots)$ accuracy schemes. This is because the leading order term of the numerical error is dispersive in even-order schemes whereas is diffusive in odd-order schemes. The electron-ion system contains the plasma waves that is a dispersive mode. Therefore, a third-order scheme would add less dispersion to the system than a second-order scheme, being more accurate and having the same reconstruction stencil.

\section{Results}

\subsection{Comparison with the classical collisionless theory}

We first study the plasma-sheath transition in a collisionless $\left(\bar{\nu}_{\mathfrak{e n}}=\bar{\nu}_{\mathfrak{i n}}=0\right)$ sheath near a planar floating wall. Since the electron inertia and the ion temperature are considered, the collisionless solution depends on three parameters: the electron-to-ion mass ratio $\varepsilon$, the ion-to-electron temperature ratio $\kappa$, and the initial normalized Debye length $\lambda$. In Fig. 2, we choose characteristic values of an argon discharge with the electrons at $T_{\mathfrak{e}}=2$ $\mathrm{eV}$ and ions at $T_{\mathfrak{i}}=0.05 \mathrm{eV}$, i.e., $\varepsilon=1.37 \cdot 10^{-5}$ and $\kappa=0.025$. We show the converged density, potential, velocity, and flux for three different domains with $L=100 \lambda_{D 0}$ (top of each panel), $300 \lambda_{D 0}$ (middle), and $1000 \lambda_{D 0}$ (bottom). The density and the flux are normalized to the density at $x=0$, that is different from the initial one. The results show only half of the domain as the solution is symmetric.

We compare the solution with the classical theory that neglects the electron inertia and the ion temperature. The solution is obtained by solving the initial-value problem (see Appendix of [9]). The classical model considers the steady mass and momentum equations for the ions, while neglecting the ion temperature, and Poisson's equation for the electric potential with Boltzmann electrons. In the classical theory, the solution depends only on the parameter

$$
q=\frac{\lambda_{c} \nu^{i z}}{u_{B}}
$$

where $\lambda_{c}$ is the Debye length at the center of the domain (that differs from the $\lambda_{D 0}$, which is the initial Debye length of the multi-fluid simulation). The parameter $q$ of the multi-fluid simulations is computed a posteriori as the $\bar{\nu}^{i z}$ is part of the solution. The three simulations correspond to $q=0.013$ in the case of $L=100 \lambda_{D 0}, q=0.0038$ $\left(L=300 \lambda_{D 0}\right)$, and $q=0.0011$ in the case of $\left(L=1000 \lambda_{D 0}\right)$. The classical theory does not need to define the length of the domain in order to obtain the solution to the 
initial-value (see e.g. [8]). For the present comparison, we set the position of the wall in the classical theory results where the ion flux equals the local electron random flux.

In Fig. 2, we compare the electron and ion densities, the electric potential, the ion velocity, and the ion flux. We recall that the classical model does not solve for the electron velocity as the electrons are computed by the Boltzmann density distribution. The multi-fluid solution agrees with the classical solution almost perfectly both in the quasi-neutral region and in the sheath for the three studied domains. One can see a small difference in the wall flux of the pressureless model that is slightly smaller. Despite this small difference that will be discussed in the following section, these results yalidate the proposed numerical scheme that solves for a complex non-linear time-dependent multi-scale system of multi-fluid equations.

In Fig. 2, we present a zoom of the solution for the density and the velocity inside the sheath. The size of the sheath is delimited with a green line that corresponds to the position where the ion velocity is equal to the modified Bohm speed. In the case of finite temperature and electron inertia $[17,12]$, this reads, in physical and normalized units,

$$
u_{B}^{m o d}=\sqrt{\frac{\mathrm{k}_{\mathrm{B}}\left(T_{\mathfrak{e}}+T_{\mathfrak{i}}\right)-m_{\mathfrak{e}} u_{\mathfrak{e}}^{2}}{m_{\mathfrak{i}}}} \text { and } \quad \bar{u}_{B}^{\text {mod }}=\sqrt{(1+\kappa)-\varepsilon \bar{u}_{\mathfrak{e}}^{2}} .
$$

This definition of the edge of the sheath results in a larger sheath for lower $q$ parameters. Nevertheless, the charge distribution as a function of the distance to the wall, measured in Debye lengths, is very similar for all the cases, as previously described by Chabert $[9]$.

A novel feature of the multi-fluid solution is that the electron velocity is selfconsistently captured by the simulation, as the electron momentum equation is solved. In the results, the ion and electron velocities are equal until the position where the ions are accelerated at the Bohm speed. An analysis of the evolution of the electron velocity in the sheath and the link with the EVDF is discussed in section 5.

\subsection{Effect of the ion temperature, the electron-to-ion mass ratio, and the elastic collisions}

In Fig. 3, we present the ion wall flux, the sheath width, and the value of the floating potential as a function of $q$ for three ion temperatures $\kappa=0.0025, \kappa=0.025$, and $\kappa=0.25$. Note that, the wall flux in our normalized units is equivalent to the usual edge-to-center density factor $h_{l}$. Additionally, we plot the sheath width by using two definitions: the position where the ions cross the modified Bohm's speed (pink), and the position where the electron density is $\bar{n}_{\mathfrak{e}}=0.9 \bar{n}_{\mathfrak{i}}$ (blue). The sheath width is measured in $\lambda_{s e}$ that is the Debye length that uses the electron density at the sheath edge. The results are systematically compared to the classical solution obtained with an initial value problem, as explained previously.

The results for $\kappa=0.025$ and 0.0025 show, in general, good agreement with the classical pressureless solution whereas the largest difference is found in the case with 
$\kappa=0.25$. The wall flux is $10 \%$ larger in the case with $\kappa=0.25$ as compared to the classical model. Similarly, the potential is around $2 \%$ smaller in the case with larger temperature, as the pressure gradient contributes to accelerate the ions to the Bohm speed. The sheath width provides similar values for the three temperatures. As seen by Chabert [9], the sheath width defined by the position of $u_{\mathrm{i}}=u_{B}^{\text {mod }}$ retrieves a larger sheath for decreasing $q$, as it was shown in Fig. 2. However, the sheath edge defined by the position where $n_{\mathfrak{e}}=0.9 n_{\mathrm{i}}$ remains at the same position for all the temperatures and values of $q$. As a matter of fact, the sheath width remains constant at a value $\approx 5 \lambda_{s e}$, as previously found by [9].

The case with $\kappa=0.25$ deviates for the main following reasons. The classical theory considers monoenergetic ions and therefore the ions are accelerated to Bohm's speed in the presheath by the electric field. However, when the temperature is taken into account, the pressure gradient contributes to this acceleration. For this reason, the plasma potential is lower in the presheath in the case where the ion temperature is considered. Inside the sheath, the pressure gradient is larger due to the steep gradient of the density. This results in a larger flux to the wall than in the case of monoenergetic ions.

In Fig. 4, we present the previous quantities for four different plasma compositions: helium $\left(\varepsilon=1.37 \cdot 10^{-4}\right)$, argon $\left(\varepsilon=1.37 \cdot 10^{-5}\right)$, krypton $\left(\varepsilon=6.5 \cdot 10^{-6}\right)$, and xenon $\left(\varepsilon=4.2 \cdot 10^{-6}\right)$. The four simulations consider $L=100 \lambda_{D 0}$ and $\kappa=0.025$. As it is known from the classical theory, the electron-to-ion mass ratio has an impact in the sheath width and the floating potential as the random flux of electrons depends on this parameter. The results show a similar wall flux for the four plasmas, which differs from the classical one that is around $1 \%$ smaller. This small difference can be attributed to the ion temperature, as shown previously. The floating potential is similar to the one retrieved by the classical theory. This small difference is also due to the ion temperature effects. The sheath width is slightly smaller for lighter plasmas in the two definitions of sheath edge. In the case of the sheath edge defined by $n_{\mathfrak{e}}=0.9 n_{\mathfrak{i}}$, we see that the classical theory retrieves a width that is slightly smaller than the multi-fluid simulations. The difference grows for increasing ion mass, which can be attributed as a small effect of the electron inertia in the sheath width. As it will be shown in section 5 , the electron velocity accelerates to approximately 0.4 times the electron thermal speed in the vicinity of the wall. The velocity gradient is larger for heavier plasmas, which can explain a larger impact in the result. Nevertheless, the difference is still a small fraction of $\lambda_{s e}$.

The influence of elastic collisions with the neutrals is shown in Figs. 5 and 6. The simulations correspond to an argon plasma with a distance between plates $L=100 \lambda_{D 0}$. We study different collisional levels in the low-pressure and intermediatepressure regime. We express the collisional frequencies as a function of the ion-neutral collisional mean-free-path $\lambda_{i}$, as follows,

$$
\bar{\nu}_{\mathfrak{i n}}=\frac{L}{\lambda_{\mathfrak{i}}} \quad \text { and } \quad \bar{\nu}_{\mathfrak{e n}}=\sqrt{\frac{T_{\mathfrak{e}}}{T_{\mathfrak{i}}}} \sqrt{\frac{m_{\mathfrak{i}}}{m_{\mathfrak{e}}}} \frac{\sigma_{\mathfrak{e n}}}{\sigma_{\mathfrak{i n}}} \frac{L}{\lambda_{\mathfrak{i}}},
$$


were the ratio between the cross sections is taken to be a characteristic value for argon, $\sigma_{\mathfrak{e n}} / \sigma_{\mathfrak{i n}}=10^{-1}$ (from [43]).

In Fig. 5 , we present the density profiles for the three collisional levels, at $\kappa=0.025$. We observe that the profile changes from the smooth collisionless profile to a cosinusoidal profile as the collisions increase. In Fig. 6, we present the ion wall flux, the sheath width, and the floating potential for $L / \lambda_{i}=0.1,1$ (both in the low-pressure limit), and 10 (intermediate-pressure). We compare the ion wall flux to the heuristic formula given by [9]. Our results show that the heuristic formula captures properly the tendency with the collisional mean-free-path. However, the practical formula underestimates the effect of the temperature both in the low-pressure and intermediate pressure regime. The sheath width computed from $n_{\mathfrak{e}}=0.9 n_{\mathfrak{i}}$ remains constant at around $5 \lambda_{s e}$. However, in the intermediate-pressure case, the Bohm speed is crossed after the space charge is created, as mentioned in [43]. Finally, the floating potential significantly increases in the intermediate pressure due to the reduction of conductivity caused by collisions.

\section{Electron fluid moments in a collisionless floating sheath}

In the previous section, we have shown that the multi-fluid model is able to selfconsistently simulate the plasma and the sheath under different conditions and for different types of ions. Furthermore, the solution for the non-linear set of equations agrees with the simplified classical theory for low ion temperatures. However, unlike the classical theory that considers Boltzmann electrons, the multi-fluid model solves the electron moment equations and, therefore, can capture effects that are consequence of the deviation from the Maxwellian distribution function, as it will be shown below. For this, we compare the multi-fluid solution to the moments derived from the kinetic solution for a collisionless sheath. By doing this, we will quantify how much an isothermal fluid solution deviates from a distribution function that is no longer isothermal.

\subsection{Analytical expressions for the electron fluid moments in a collisionless floating} sheath

The analytical solution for the EVDF inside a collisionless steady-state sheath can be found with the theory of characteristics $[34,10]$. We summarize here the results that are shown in the mentioned works. We consider the Vlasov equation in one dimension at stationary state for the electron distribution function inside a collisionless sheath under the influence of the electric potential, as follows

$$
v_{x} \frac{\partial f_{\mathfrak{e}}}{\partial x}+\frac{e}{m_{\mathfrak{e}}} \frac{\partial \phi}{\partial x} \frac{\partial f_{\mathfrak{e}}}{\partial v_{x}}=0
$$

with the boundary condition that assumes that the wall collects all the incident electrons

$$
f_{\mathfrak{e}}\left(x=x_{\text {wall }}, v_{x}\right)=0 \quad \text { for } \quad v_{x}<0 .
$$


We consider the $x$-direction that grows from the sheath edge towards the wall. The equation can be solved by the method of characteristics in the phase space $\left(x, v_{x}\right)$. Along the characteristic curve $V(x)$ of the phase space, the derivative in space of the distribution function is

$$
\frac{d}{d x} f_{\mathfrak{e}}(x, V(x))=\frac{\partial f_{\mathfrak{e}}}{\partial x}+V^{\prime} \frac{\partial f_{\mathfrak{e}}}{\partial v_{x}}=\frac{\partial f_{\mathfrak{e}}}{\partial x}+\frac{e}{m_{\mathfrak{e}} V} \frac{\partial \phi}{\partial x} \frac{\partial f_{\mathfrak{e}}}{\partial v_{x}}=0
$$

Consequently, Eq. (19) reduces to a trivial ODE along the characteristic line, $\frac{d}{d x} F_{\mathfrak{e}}(x)=$ 0 , where $F_{\mathfrak{e}}(x)=f_{\mathfrak{e}}(x, V(x))$.

The equation for the characteristic curve $V(x)$ is found in Eq. (21), as follows

$$
V^{\prime}=\frac{e}{m_{\mathfrak{e}} V} \frac{\partial \phi}{\partial x}, \quad \text { thus } \quad \frac{V^{2}(x)}{2}-\frac{e}{m_{\mathfrak{e}}} \phi(x)=\text { const. }
$$

This relation defines the family of curves in the phase space along which the distribution function is constant. We can observe that due to the boundary condition of Eq. (20), the phase space is divided into two subdomains, as shown in Fig. 7. As the wall does not emit electrons, the distribution function is zero for velocities that are $v_{x}<-\sqrt{2 e \phi / m_{\mathfrak{e}}}$, which is the characteristic curve that touches the $x=x_{\text {wall }}$ at $v_{x}=0$. We recall that the potential at the wall is set to zero.

As the distribution function is constant along these characteristics in the phase space, by knowing the solution in the sheath edge we can obtain the distribution function inside the sheath. We assume that the distribution function at the sheath edge is a Maxwellian with density $n_{\mathfrak{e}_{s h}}$ and temperature $T_{\mathfrak{c}_{s h}}$, as follows

$$
f_{\mathfrak{e}}\left(x_{s h}, v_{x}\right) \equiv f_{\mathfrak{e}_{s h}}\left(v_{x}\right)=n_{\mathfrak{e}_{s h}}\left(\frac{m_{\mathfrak{e}}}{2 \pi \mathrm{k}_{\mathrm{B}} T_{\mathfrak{e}_{s h}}}\right)^{1 / 2} \exp \left(-\frac{m_{\mathfrak{e}} v_{x}^{2}}{2 \mathrm{k}_{\mathrm{B}} T_{\mathfrak{e}_{s h}}}\right) .
$$

Here, the subscript $s h$ is used for the value of the variables at the edge of the sheath. This distribution function will have a truncated tail for $v_{x}<-\sqrt{2 e \phi_{s h} / m_{\mathfrak{e}}}$, as explained before. We have considered a one-dimensional Maxwellian distribution function since, in a planar sheath, the distribution function only changes in this direction and remains unmodified in the other velocity spaces. Finally, since the value of the distribution function is constant on each characteristic, we can conclude that

$$
f_{\mathfrak{e}}\left(x, v_{x}\right)=f_{\mathfrak{e}_{s h}}\left(\sqrt{v_{x}^{2}-\frac{2 e}{m_{\mathfrak{e}}}\left(\phi-\phi_{s h}\right)}\right) .
$$

Consequently, the distribution function reads

$$
f_{\mathfrak{e}}\left(x, v_{x}\right)= \begin{cases}n_{\mathfrak{e}_{s h}}\left(\frac{m_{\mathfrak{e}}}{2 \pi \mathrm{k}_{\mathrm{B}} T_{\mathfrak{e}_{s h}}}\right)^{1 / 2} \exp \left(-\frac{m_{\mathfrak{e}} v_{x}^{2}}{2 \mathrm{k}_{\mathrm{B}} T_{\mathfrak{e}_{s h}}}+\frac{e\left(\phi-\phi_{s h}\right)}{\mathrm{k}_{\mathrm{B}} T_{\mathfrak{e}_{s h}}}\right) & \text { if } v_{x} \geq-\sqrt{\frac{2 e \phi}{m_{\mathfrak{e}}}} \\ 0 & \text { otherwise. }\end{cases}
$$

The same distribution function is used in [44]. In normalized units, the distribution function reads

$$
\bar{f}_{\mathfrak{e}}\left(\bar{x}, \bar{v}_{x}\right)= \begin{cases}\bar{n}_{\mathfrak{e}_{s h}}\left(\frac{\varepsilon}{2 \pi}\right)^{1 / 2} \exp \left(-\frac{\varepsilon \bar{v}_{x}^{2}}{2}+\left(\bar{\phi}-\bar{\phi}_{s h}\right)\right) & \text { if } \bar{v}_{x} \geq-\sqrt{\frac{2 \bar{\phi}}{\varepsilon}} \\ 0 & \text { otherwise }\end{cases}
$$


Note that since the electron temperature is constant, the electron temperature at the sheath edge is equal to the reference temperature.

The previous distribution function is illustrated in Fig. 7. For these results, we use the electric potential calculated in the simulation with $q=0.013, \varepsilon=1.37 \cdot 10^{-5}$, and $\kappa=0.025$. The figure shows a distribution function that is truncated in the velocity space, as previously explained in $[34,10]$.

We compute the moments of the truncated EVDF in order to compare them to the solution of the multi-fluid code inside the sheath. The zeroth-order moment corresponds to the electron density inside the sheath, that reads

$$
\bar{n}_{\mathfrak{e}}=\int_{-\infty}^{+\infty} \bar{f}_{\mathfrak{e}} d \bar{v}_{x}=\bar{n}_{\mathfrak{e}_{s h}} F(\bar{\phi}) e^{\left(\bar{\phi}-\bar{\phi}_{s h}\right)} \quad \text { with } \quad F(\bar{\phi})=\left(1-\frac{1}{2} \operatorname{erfc}(\sqrt{\bar{\phi}})\right)^{\prime} .
$$

As the reader may notice, the electron density follows a distribution that is very close to the classical Boltzmann distribution but it is modulated by the factor $F(\bar{\phi})$. This factor varies from 1 (at the sheath edge) to $1 / 2$ (at the wall), decreasing rapidly when the potential is close to zero, i.e., in the vicinity of the wall. As it will be shown below, the variation of factor depends on the mass ratio between electrons and ions, as the potential drop depends on this parameter.

The first-order moment corresponds to the particle flux that reads as follows

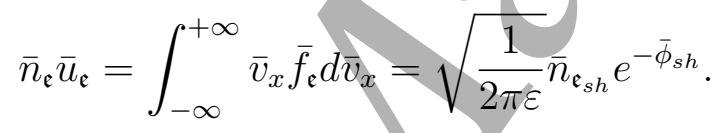

The particle flux is constant inside the sheath and the expression is identical to the classical theory. From the flux and the density, one can obtain the evolution of the velocity inside the sheath, as follows,

$$
\bar{u}_{\mathfrak{e}}=\sqrt{\frac{1}{2 \pi \varepsilon}} \frac{e^{-\bar{\phi}}}{F(\bar{\phi})} .
$$

This acceleration inside the sheath is a consequence of the truncation of the distribution function. In this case, âs no electrons are reflected from the wall, the negative part of the distribution function is cut as it approaches the wall. As a consequence of this truncation, there is a larger number of particles with positive velocity, which results in a macroscopic positive velocity. We highlight that, in this case, the macroscopic velocity does not indicate that the distribution function is a drifting Maxwellian.

The pressure in the $x$-direction can be derived from the following moment of the distribution function, as follows,

$$
\bar{p}_{\mathfrak{e}_{x}}=\varepsilon \int_{-\infty}^{+\infty} \bar{v}_{x}^{2} \bar{f}_{\mathfrak{e}} d \bar{v}_{x}-\varepsilon \bar{u}_{\mathfrak{e}}^{2}=\bar{n}_{\mathfrak{e}_{s h}} e^{\left(\bar{\phi}-\bar{\phi}_{s h}\right)} \frac{\left(4 \pi F(\bar{\phi})^{2}-4 \sqrt{\pi \bar{\phi}} e^{-\bar{\phi}} F(\bar{\phi})-2 e^{-2 \bar{\phi}}\right)}{4 \pi F(\bar{\phi})} .
$$

As it occurs with the expression for the density, the classical expression for pressure is multiplied by a factor that tends to one at the edge of the sheath and to $(\pi-2) / 2 \pi \approx$ 
0.181 at the wall. Note that the pressure is taken in the $x$-direction and due to the truncation of the distribution function, the pressure is anisotropic.

Finally, the temperature in the $x$-direction inside the sheath is defined as

$$
\bar{T}_{\mathfrak{e}_{x}}=\frac{\bar{p}_{\mathfrak{e}_{x}}}{\bar{n}_{\mathfrak{e}}}=\frac{4 \pi F(\bar{\phi})^{2}-4 \sqrt{\pi \bar{\phi}} e^{-\bar{\phi}} F(\bar{\phi})-2 e^{-2 \bar{\phi}}}{4 \pi F(\bar{\phi})^{2}} .
$$

This function tends to one at the edge of the sheath whereas it is $(\pi-2) / \pi \approx 0.36$ at the wall. Note that the evolution of the potential inside the sheath depends on the mass ratio between the electrons and ions. However, the values at the sheath edge and at the wall do not depend on the composition of the gas. This expression for the temperature inside the sheath, that is rigorously derived from the kinetic theory, shows that the isothermal assumption for the electrons is in contradiction with the kinetic theory. Furthermore, it is in agreement with the PIC simulations [10] that show a decrease of the electron temperature normal to the wall. Similarly, the numerical valyes proposed by the present model are in quantitative agreement with the kinetic simulations of [12] (see profile of $\bar{T}_{\mathfrak{e}_{x}}$ in Fig. 8 of [12]). Note that the reference temperature of the present model is the temperature at the sheath edge, therefore, the temperature at the wall is $T_{\mathfrak{e}_{x}}=0.36 T_{x_{\mathrm{e}}}^{s e}$. The expressions developed in this work are similar to those of [44]. However, in this work, we have taken into account the anisotropy of the temperature inside the sheath.

\subsection{Comparison between the kinetic theory and the multi-fluid solution}

In Fig. 8, we show the comparison between the multi-fluid solution of a xenon, argon, and helium plasma with $\kappa=0.025$ and $L=1000 \lambda_{D 0}$ and the moments of the truncated Maxwellian inside a collisionless sheath (Eqs. (27), (29),(30),(31)). The moments depend on the potential that is taken from the multi-fluid simulation.

The multi-fluid solution for the electron dynamics shows a good agreement with the analytical moments for the density, the velocity, and the pressure. As a result, we can conclude that the multi-fluid solution is compatible with a truncated Maxwellian EVDF inside the sheath. Furthermore, the electron inertial terms that are seen to have very small impact in the global parameters of the solution, are fundamental in order to link the fluid description with the kinetic theory in the sheath.

In our simulations as well as in the classical theory, the electron fluid is assumed to be isothermal. However, the analytical expression for the temperature shows that the electron temperature decreases in the last Debye lengths close to the wall. This discrepancy causes a small difference between the analytical and the multi-fluid solutions close to the wall. The analytical temperature variation strongly depends on the electronto-ion mass ratio, as shown in Fig. 8. Nevertheless, the values of the temperature at the wall and at the sheath edge are the same for all species. As previously seen in PIC simulations [10], the decrease of electron energy inside the sheath can result in a decrease of the floating potential. For this reason, higher moments of the electron distribution function need to be considered by multi-fluid models in order to achieve a solution that is fully in agreement with the kinetic theory. 


\section{Conclusions}

We present high-order accuracy high-resolution multi-fluid simulations of the plasmasheath transition of a planar floating wall in a plasma composed by positive ions and electrons under low pressure conditions. As opposed to the classical theory, the multi-fluid simulations consider the electron inertial terms and the effect of a finite ion temperature. Nevertheless, the resulting system of equations is very stiff due to the multi-scale character of the solution, especially when the realistic electron mass is considered. Additionally, the solution contains sharp gradients and speeds that range from zero to supersonic values. The system is therefore difficult to solve numerically and demands high-order accuracy numerical schemes in order to reduce the error of the numerical discretization. In this paper, we have proposed a numerical procedure that retrieves a steady-state solution by solving the time dependent non-linear multifluid equations to steady state. We have shown the importance of the accuracy of the discretization and the resolution as well as consistent boundary conditions in order to obtain meaningful results.

We have benchmarked the multi-fluid solution against the simplified classical solution for different temperatures, electron-to-ion/mass ratio, and collisional levels. The collisionless solution at low temperature shows perfect agreement with the classical solution, which demonstrates the accuracy and consistency of the proposed numerical scheme and boundary conditions. Nevertheless, the multi-fluid solver is able to find a general solution for ion finite temperatures and different levels of collisionality. The temperature of the ions in a discharge is typically of the order 100 times lower than the electrons. However, as shown by Meige et al. [45], the ions can increase their temperature in the direction perpendicular to their motion through the presheath. Moreover, in other plasma applications, such as magnetically-confined fusion, the ion temperature can be comparable to the electrons. For this, reason, a fluid model that considers the ion temperature can be regarded as a development of the classical theory. Our solution shows that under collisionless conditions, the impact of the ion temperature is only significant when the ion temperature is a large fraction of the electron temperature, i.e., $T_{\mathfrak{i}} \approx 0.25 T_{\mathfrak{e}}$. Simflarly, the combination of the ion-temperature effects and the elastic collisions show a small difference up to $10 \%$ with the heuristic formula for the edge-to-center density factor proposed by [43]. On the other hand, the effect of the electron inertial terms has a negligible impact in the global parameters of the discharge, as expected. Nevertheless, the electron inertial terms are found to be fundamental in order to link the fluid solution with the shape of the EVDF inside the sheath under collisionless conditions.

In this paper, we have derived the expressions of the electron fluid quantities inside a steady-state collisionless sheath from the kinetic theory by assuming a truncated Maxwellian EVDF with a wall that absorbs the electron random flux. With these assumptions, we show that the electron temperature is no longer constant inside the sheath, as recently shown in PIC simulations [10]. The multi-fluid solution shows good 
agreement with the analytical formulas derived from the kinetic theory for the electron density, the velocity, and the pressure.

In this paper, we have demonstrated that multi-fluid solvers are able to selfconsistently capture the plasma-sheath transition under collisionless and low-pressure conditions. Even though the classical fluid theory works fine with cold ions, we show that a solution that is beyond the Boltzmann electrons and mono-energetic ions can generalize the classical theory for conditions where the ions have a finite temperature. The solution of the electron dynamics, while keeping the electron inertia, can capture phenomena that is beyond the drift-diffusion approximation, especially under weakly collisional conditions. Multi-fluid models can be of especial interest for plasmas with strong electron drifts where electron inertial term is no longer negligible. These large drifts can be created, for instance, by a magnetic field. The present work can be interesting for those simulations, while consistently including the sheaths in the numerical domain. Despite the numerical difficulties of multi-fluid models, we have demonstrated that a high-order numerical scheme and the proposed boundary conditions, the simulation is able capture the plasma-sheath transition, being consistent with both the classical and kinetic theories. This work can be extended to more complex multi-fluid models that consider higher-order moments, including non-isotropic tensor [46] which can lead to instabilities at high-temperature sheaths [47].

\section{Appendix A. Numerical method}

We propose to solve the system of equations (7)-(11) by means of a finite volume method for the fluid equations and a finite difference method for the Poisson equation. We detail here the proposed numerical scheme that is implemented in a single dimension and uses the Roe scheme for the convective fluxes with third-order total variation diminishing (TVD) reconstruction, Thomas's algorithm for Poisson's equation, and a third-order TVD Runge-Kutta scheme for the time integration.

In the following, we write the fluid equations, i.e., eqs. (7)-(10) in conservation form as

$$
\frac{\partial}{\partial \bar{t}} \bar{U}+\frac{\partial}{\partial \bar{x}} \bar{F}=\bar{S}
$$

with the conservative variable vector

$$
\bar{U}=\bar{U}(\bar{x}, \bar{t})=\left(\bar{n}_{\mathfrak{e}}, \bar{n}_{\mathfrak{e}} \bar{u}_{\mathfrak{e}}, \bar{n}_{\mathfrak{i}}, \bar{n}_{\mathfrak{i}} \bar{u}_{\mathfrak{i}}\right)^{T}
$$

the flux vector

$$
\bar{F}=\bar{F}(\bar{U})=\left(\bar{n}_{\mathfrak{e}} \bar{u}_{\mathfrak{e}}, \bar{n}_{\mathfrak{e}}\left(\bar{u}_{\mathfrak{e}}^{2}+\varepsilon^{-1}\right), \bar{n}_{\mathfrak{i}} \bar{u}_{i}, \bar{n}_{\mathfrak{i}}\left(\bar{u}_{i}^{2}+\kappa\right)\right)^{T}
$$

and the source term vector

$$
\bar{S}=\bar{S}(\bar{U}, \bar{\phi})=\left(\bar{n}_{\mathfrak{e}} \bar{\nu}^{i z}, \bar{n}_{\mathfrak{e}} \varepsilon^{-1} \frac{\partial}{\partial \bar{x}} \bar{\phi}-\bar{\nu}_{\mathfrak{e n}} \bar{n}_{\mathfrak{e}} \bar{u}_{\mathfrak{e}}, \bar{n}_{\mathfrak{e}} \bar{\nu}^{i z}, \quad-\bar{n}_{\mathfrak{i}} \frac{\partial}{\partial \bar{x}} \bar{\phi}-\bar{\nu}_{\mathfrak{i n}} \bar{n}_{\mathfrak{i}} \bar{u}_{\mathfrak{i}}\right)^{T}
$$


The importance of writing the equations in conservation form, as opposed to other methods that solve for other non-conserved magnitudes such as velocity and temperature, is fundamental for finite volume methods. Solving for conservative variables helps to preserve the conservation laws in the discrete solution of finite volume schemes as well as the convergence in the representation of the weak solution (discontinuities) of the system (see 12.9 of [48]).

\section{Appendix A.1. Finite volume scheme with Roe flux}

The domain $x \in[-L / 2, L / 2]$ is divided into $N$ elements of equal length $\Delta x$. We write the integral form of Eq. (A.1) for each element $i \in N$ as

$$
\frac{d}{d t} \int_{\Omega_{i}} \bar{U} d \Omega+\oint_{\partial \Omega_{i}} \bar{F} \cdot \vec{n} d \sigma=\int_{\Omega_{i}} \bar{S} d \Omega
$$

We approximate the value of $\bar{U}$ as a piecewise function inside the volume $\Omega_{i}$ that we denote as $\mathbf{U}_{i}$. The flux on the interfaces, $\partial \Omega_{i}$ (with outward normal $\vec{n}$ ), is approximated by a numerical flux function $\mathcal{F}\left(\mathbf{U}_{L}, \mathbf{U}_{R}\right)$ that is in general a function of the values of the variables on the right and left of the cell interface. The source is approximated by a piecewise constant value denoted as $\mathbf{S}_{i}$. After making these definitions, the resulting finite volume discretization for the cell $i$ reads

$$
\frac{d}{d t} \mathbf{U}_{i}+\frac{1}{\Delta x}\left(\mathbf{F}_{i+1 / 2}-\mathbf{F}_{i-1 / 2}\right)=\mathbf{S}_{i}
$$

where $\mathbf{F}_{i+1 / 2}=\mathcal{F}\left(\mathbf{U}_{L}, \mathbf{U}_{R}\right), \mathbf{U}_{L / R}$ are the reconstructed values at the left and right of the cell interface $i+1 / 2$.

In order to define the numerical flux function, we first write the convective flux as the contribution of ions and electrons $\bar{F}(\bar{U})=\left(\bar{F}^{\mathfrak{e}}\left(\bar{U}^{\mathfrak{e}}\right), \bar{F}^{\mathfrak{i}}\left(\bar{U}^{\mathfrak{i}}\right)\right)^{T}$, where

$$
\bar{F}^{\alpha}\left(\bar{U}^{\alpha}\right)=\left(\bar{n}_{\alpha} \bar{u}_{\alpha}, \bar{n}_{\alpha}\left(\bar{u}_{\alpha}^{2}+c_{\alpha}^{2}\right)\right)^{T} \quad \text { with } \quad \alpha \in\{\mathfrak{e}, \mathfrak{i}\} .
$$

We use the same flux scheme $\mathcal{F}\left(\mathbf{U}_{L}, \mathbf{U}_{R}\right)$ for ions and electrons: the Roe scheme [37] that reads

$$
\mathbf{F}_{i+1 / 2}^{\alpha}=\frac{1}{2}\left(\bar{F}^{\alpha}\left(\mathbf{U}^{L}\right)+\bar{F}^{\alpha}\left(\mathbf{U}^{R}\right)\right)-\frac{1}{2} \sum_{p=1}^{2}\left|\lambda_{p}\right| \alpha_{p} \mathbf{r}_{p} .
$$

The first term is a central discretization whereas the second is the so-called numerical dissipation that makes the scheme monotonic (non-oscillatory). The eigenvalues at the interface are $\lambda_{1}=\hat{u}_{\alpha}-c_{\alpha}$ and $\lambda_{2}=\hat{u}_{\alpha}+c_{\alpha}$. We recall that the speeds of sound for the electrons and ions are $c_{\mathfrak{e}}^{2}=\varepsilon^{-1}$ and $c_{\mathfrak{i}}^{2}=\kappa$, respectively. The Roe-average velocity is defined as

$$
\hat{u}_{\alpha}=\frac{\bar{n}_{\alpha}^{R} \bar{u}_{\alpha}^{R}+\bar{n}_{\alpha}^{L} \bar{u}_{\alpha}^{L}}{\bar{n}_{\alpha}^{R}+\bar{n}_{\alpha}^{L}}
$$

The right eigenvectors are

$$
\mathbf{r}_{1}=\left(\begin{array}{c}
1 \\
\hat{u}_{\alpha}-c_{\alpha}
\end{array}\right), \text { and } \mathbf{r}_{2}=\left(\begin{array}{c}
1 \\
\hat{u}_{\alpha}+c_{\alpha}
\end{array}\right)
$$


The coefficients $\alpha_{p}$ are defined as

$$
\alpha_{p}=\mathbf{r}^{p} \cdot\left(\mathbf{U}_{R}^{\alpha}-\mathbf{U}_{L}^{\alpha}\right) \quad \text { with } \mathbf{r}^{1}=\frac{1}{2 c_{\alpha}}\left(\hat{u}_{\alpha}-c_{\alpha},-1\right), \quad \text { and } \mathbf{r}^{2}=\frac{1}{2 c_{\alpha}}\left(-\hat{u}_{\alpha}+c_{\alpha}, 1\right) \text {. }
$$

Third order accuracy in space is achieved with a TVD MUSCL (Monotonic Upwind Scheme for Conservation Laws) scheme [38] that is using flux limiter proposed by [40].

We use the method of lines that separates the space and time integrations. Consequently, after the computation of the fluxes, as explained above, we obtain an ODE in time, as follows

$$
\frac{d}{d t} \mathbf{U}_{i}=\mathbf{R}_{i}, \quad \text { with } \quad \mathbf{R}_{i}^{n}=-\frac{1}{\Delta x}\left(\mathbf{F}_{i+1 / 2}-\mathbf{F}_{i-1 / 2}\right)+\mathbf{S}_{i}
$$

This ODE is resolved with a third-order TVD Runge Kutta scheme, which, as proposed by [41], reads

$$
\begin{aligned}
& \mathbf{U}_{i}^{(1)}=\mathbf{U}_{i}^{n}+\frac{\Delta t}{\Delta x} \mathbf{R}_{i}^{n}, \\
& \mathbf{U}_{i}^{(2)}=\frac{3}{4} \mathbf{U}_{i}^{n}+\frac{1}{4} \mathbf{U}_{i}^{(1)}+\frac{1}{4} \frac{\Delta t}{\Delta x} \mathbf{R}_{i}^{(1)} \\
& \mathbf{U}_{i}^{n+1}=\frac{1}{3} \mathbf{U}_{i}^{n}+\frac{2}{3} \mathbf{U}_{i}^{(2)}+\frac{2}{3} \frac{\Delta t}{\Delta x} \mathbf{R}_{i}^{(2)}
\end{aligned}
$$

The numerical scheme has a Courant-Friedrichs-Lewy (CFL) condition for numerical stability, as explained in Eq. (15).

The Poisson equation for the electric potential is solved at the end of each time-step through Thomas' algorithm.

In order to impose the boundary conditions, we use the ghost cell approach. Accordingly, we add one cell of size $\Delta x$ adjacent to the boundary at both sides of the domain, i.e., $\mathbf{U}_{0}$ and $\mathbf{U}_{N+1}$. We don't solve the equations in those cells, but we imposed their values at every time step, so the flux at the interface of the cell, i.e., $\mathbf{F}_{1 / 2}$ and $\mathbf{F}_{N+1 / 2}$ can be calculated. As shown in Eq. (13), the value that we want to impose is the electron flux. Nevertheless, we still need to fulfil the continuity equation (the gradient of the flux is zero at the wall). Therefore, we impose the velocity at the ghost cell through a linear extrapolation as

$$
\left.\bar{u}_{\mathfrak{e}}\right|_{G}=\left.2 \bar{u}_{\mathfrak{e}}\right|_{\text {wall }}-\left.\bar{u}_{\mathfrak{e}}\right|_{I} \quad \text { with }\left.\quad \bar{u}_{\mathfrak{e}}\right|_{\text {wall }}=\sqrt{\frac{1}{2 \pi \varepsilon}}
$$

where the subscript $I$ stands for the cell in contact with the boundary inside the domain and $G$ for the ghost cell.

Therefore, the variables at the ghost cell are imposed as

$$
\left(\begin{array}{c}
\left.\bar{n}_{\mathfrak{e}}\right|_{G} \\
\left.\left(\bar{n}_{\mathfrak{e}} \bar{u}_{\mathfrak{e}}\right)\right|_{G} \\
\left.\bar{n}_{\mathfrak{i}}\right|_{G} \\
\left.\left(\bar{n}_{\mathfrak{i}} \bar{u}_{\mathfrak{i}}\right)\right|_{G}
\end{array}\right)=\left(\begin{array}{c}
\left.\left(\bar{n}_{\mathfrak{e}} \bar{u}_{\mathfrak{e}}\right)\right|_{I} /\left.\bar{u}_{\mathfrak{e}}\right|_{G} \\
\left.\left(\bar{n}_{\mathfrak{e}} \bar{u}_{\mathfrak{e}}\right)\right|_{I} \\
\left.\bar{n}_{\mathfrak{i}}\right|_{I} \\
\left.\left(\bar{n}_{\mathfrak{i}} \bar{u}_{\mathfrak{i}}\right)\right|_{I}
\end{array}\right) .
$$

\section{Acknowledgements}

A.A.L. has been funded by the postdoctoral fellowship from the Fondation Mathematique Jacques Hadamard (FMJH), LabEx Mathématique Hadamard - ANR11-LABX-0056 
and LabEX LASIPS - ANR10-LABX-0032 and the project POSEIDON supported by ANR ( n ANR-16-CHIN-003-01) and Safran Aicraft Engines. T. M. is supported by a "Chaire Jean d'Alembert" of University Paris Saclay at Ecole Polytechnique.

\section{References}

[1] Post D E and Behrisch R 1984 Physics of plasma-wall interactions in controlled fusion (United States: Plenum Press) URL http://inis.iaea.org/search/search.aspx?orig_q= $\mathrm{RN}: 18053918$

[2] Liebermann M A and Lichtenberg A J 1995 Principles of plasma discharges and materials processing. (New York: John Wiley \& Sons, Inc.) (Preprint https://onlinelibrary.wiley. com/doi/pdf/10.1002/maco.19950460909) URL https://onlinelibrary.wiley.com/doi/ abs/10.1002/maco.19950460909

[3] Hutchinson I H 2002 Principles of Plasma Diagnostics (Cambridge: Cambridge University Press) ISBN 9780521675741 URL https://www cambridge. org/core/books/ principles-of-plasma-diagnostics/93578947341481B0F9169D598D535E76

[4] Heberlein J, Pfender E and Mentel J 2010 Journal of Physics D, Applied Physics 4331 URL http://dx.doi.org/10.1088/0022-3727/43/2/023001

[5] Ahedo E, Gallardo J M and Martínez-Sánchez M 2003 Physics of Plasmas 10 3397-3409 (Preprint https://doi.org/10.1063/1.1584432) URL https://doi.org/10.1063/1.1584432

[6] Tonks L and Langmuir I 1929 Physical Review 33(2) 195-210 URL https://link.aps.org/doi/ 10.1103/PhysRev.33.195

[7] Bohm D 1949 The characteristics of electrical discharges in magnetic fields (New York: MacGrawHill) chap 3, p 77

[8] Riemann K U, Seebacher J, Sr D D T and Kuhn S 2005 Plasma Physics and Controlled Fusion 471949 URL http://stacks.iop.org/0741-3335/47/i=11/a=006

[9] Chabert P 2014 Plasma Sources Science and Technology 23065042 URL https://doi.org/10. $1088 \% 2 \mathrm{~F} 0963-0252 \% 2 \mathrm{~F} 23 \% 2 \mathrm{~F} 6 \% 2 \mathrm{~F} 065042$

[10] Tavant A, Lucken R, Bourdon A and Chabert P 2019 Plasma Sources Science and Technology 28 075007 URL https://doi.org/10.1088\%2F1361-6595\%2Fab279b

[11] Cagas P 2018 Continuum Kinetic Simulations of Plasma Sheaths and Instabilities Ph.D. thesis Virginia Tech

[12] Cagas P, Hakim A, Juno J and Srinivasan B 2017 Physics of Plasmas 24022118 URL https: //doi.org/10.1063/1.4976544

[13] Kolobov V and Arslanbekoy R 2003 Microelectronic Engineering 69606 - 615 ISSN 01679317 proceedings of the Symposium and Summer School on: Nano and Giga Challenges in Microelectronics Research and Opportunities in Russia URL http://www . sciencedirect. com/ science/article/pii/S0167931703003526

[14] Robertson S 2013 Plasma Physics and Controlled Fusion 55093001 URL https://doi.org/10. $1088 \% 2 \mathrm{~F} 0741-3335 \% 2 \mathrm{~F} 55 \% 2 \mathrm{~F} 9 \% 2 \mathrm{~F} 093001$

[15] Hofer R, Mikellides I, Katz I and Goebel D 2007 Wall sheath and electron mobility modeling in hybrid-pic hall thruster simulations 43rd AIAA/ASME/SAE/ASEE Joint Propulsion Conference \&3 Exhibit) (Preprint https://arc.aiaa.org/doi/pdf/10.2514/6.2007-5267) URL https://arc .aiaa.org/doi/abs/10.2514/6.2007-5267

[16] Loizu J, Ricci P, Halpern F D and Jolliet S 2012 Physics of Plasmas 19122307 URL https: //doi.org/10.1063/1.4771573

[17] Scheiner B, Baalrud S D, Yee B T, Hopkins M M and Barnat E V 2015 Physics of Plasmas 22 123520 (Preprint https://doi.org/10.1063/1.4939024) URL https://doi.org/10.1063/1. 4939024 
[18] Yee B T, Scheiner B, Baalrud S D, Barnat E V and Hopkins M M 2017 Plasma Sources Science and Technology 26025009 URL https://doi.org/10.1088\%2F1361-6595\%2Faa56d7

[19] Borgohain B and Bailung H 2017 Physics of Plasmas 24113512 (Preprint https://doi .org/10. 1063/1.5006133) URL https://doi.org/10.1063/1.5006133

[20] Stenzel R L, Gruenwald J, Ionita C and Schrittwieser R 2011 Physics of Plasmas 18 062113 (Preprint https://doi.org/10.1063/1.3601860) URL https://doi.org/10.1063/1. 3601860

[21] Guittienne P, Howling A A and Furno I 2018 Physics of Plasmas 25093519 (Preprint https: //doi.org/10.1063/1.5041496) URL https://doi.org/10.1063/1.5041496

[22] Andrés N, Martin L, Dmitruk P and Gómez D 2014 Physics of Plasmas 21072904 (Preprint https://doi.org/10.1063/1.4890021) URL https://doi.org/10.1063/1.4890021

[23] Jana S, Ghosh S and Chakrabarti N 2017 Physics of Plasmas 24102307 (Preprint https: //doi.org/10.1063/1.4994118) URL https://doi.org/10.1063/1.4994118

[24] Srinivasan B and Hakim A 2018 Physics of Plasmas 25092108 (Preprint https://doi.org/10. 1063/1.5046098) URL https://doi.org/10.1063/1.5046098

[25] Smolyakov A I, Chapurin O, Frias W, Koshkarov O, Romadanov I, Tang T, Umansky M, Raitses Y, Kaganovich I D and Lakhin V P 2016 Plasma Physics and Controlled Fusion 59014041 URL https://doi.org/10.1088\%2F0741-3335\%2F59\%2F1\%2F014041

[26] Ballesteros J, Fernández Palop J I, Hernández M A and Morales Crespo R 2006 Applied Physics Letters 89101501 (Preprint https://doi.org/10.1063/1.2345252) URL https://doi.org/ $10.1063 / 1.2345252$

[27] Morales Crespo R 2018 Physics of Plasmas 25063509 (Preprint https://doi.org/10.1063/1. 5025828) URL https://doi.org/10.1063/1.5025828

[28] Shumlak U and Loverich J 2003 Journal of Computational Physics 187 620-638 ISSN 0021-9991 URL http://dx.doi.org/10.1016/S0021-9991(03)00151-7

[29] Hakim A, Loverich J and Shumlak U 2006 Journal of Computational Physics 219 418-442 ISSN 0021-9991 URL http://dx.doi.org/10.1016/j.jcp.2006.03.036

[30] Alvarez Laguna A, Ozak N, Lani A, Deconinck H and Poedts S 2018 Computer Physics Communications 23131 - 44 ISSN 0010-4655 URL http://www. sciencedirect.com/science/ article/pii/S0010465518301590

[31] Alvarez Laguna A, Pichard T, Magin T, Chabert P, Bourdon A and Massot M 2019 (Submitted to Journal of Computational Physics) arXiv:1904.13092 (Preprint 1904.13092)

[32] Godyak V A 1982 Physics Letters A 89 80-81 URL http://www.sciencedirect.com/science/ article/pii/0375960182905102

[33] Kaganovich I D 2002 Physics of Plasmas 9 4788-4793 URL https://doi.org/10.1063/1. 1515274

[34] Badsi M, Campos Pinto M and Després B 2016 Kinetic and Related Models 9 URL https: //hal.archives-ouvertes.fr/hal-01075646

[35] Dubois F and Floch P L 1988 Journal of Differential Equations 7193 - 122 ISSN 0022-0396 URL http://www.sciencedirect.com/science/article/pii/002203968890040X

[36] Godlewski E and Raviart P A 1996 Numerical Approximation of Hyperbolic Systems of Conservation Laws (Applied Mathematical Sciences Book Series vol 118) (New York: Springer)

[37] Roe P 1997 Journal of Computational Physics 135250 - 258 ISSN 0021-9991 URL http: //www.sciencedirect /com/science/article/pii/S0021999197957053

[38] van Leer B 1979 Journal of Computational Physics 32101 - 136 ISSN 0021-9991 URL http: //www.sciencedirect.com/science/article/pii/0021999179901451

[39] Harten A 1983 Journal of Computational Physics 49357 - 393 ISSN 0021-9991 URL http: //www.sciencedirect.com/science/article/pii/0021999183901365

[40] Čada M and Torrilhon M 2009 Journal of Computational Physics 2284118 - 4145 ISSN 0021-9991 URL http://www.sciencedirect.com/science/article/pii/S0021999109000953

[41] Gottlieb S and Shu C W 1998 Mathematics of Computation 67 73-85 ISSN 0025-5718 URL 
http://dx.doi.org/10.1090/S0025-5718-98-00913-2

[42] Degond P, Liu J and Vignal M H 2008 SIAM Journal on Numerical Analysis 46 1298-1322 URL https://doi.org/10.1137/070690584

[43] Chabert P and Braithwhaite N 2011 Physics of radio-frequency plasmas (Cambridge: Cambridge University Press)

[44] Baalrud S D and Hegna C C 2011 Plasma Sources Science and Technology 20025013 URL https : //doi .org/10.1088\%2F0963-0252\%2F20\%2F2\%2F025013

[45] Meige A, Sutherland O, Smith H B and Boswell R W 2007 Physics of Plasmas 14032104 (Preprint https://doi.org/10.1063/1.2709648) URL https://doi.org/10.1063/1.2709648

[46] Miura K and Groth C 2007 Development of two-fluid magnetohydrodynamics model for nonequilibrium anisotropic plasma flows 38th Plasmadynamics and Lasers Conference (American Institute of Aeronautics and Astronautics) URL https://doi.org/10.2514/6.2007-4373

[47] Tang X Z 2011 Plasma Physics and Controlled Fusion 53082002 URL https://doi.org/10. $1088 \% 2 \mathrm{~F} 0741-3335 \% 2 \mathrm{~F} 53 \% 2 \mathrm{~F} 8 \% 2 \mathrm{~F} 082002$

[48] LeVeque R 2002 Finite Volume Methods for Hyperbolic Problems Cambridge Texts in Applied Mathematics (Cambridge: Cambridge University Press) ISBN 9781139434188 URL https: //books.google.fr/books?id=mfAfAwAAQBAJ 



Figure 2. Comparison between the multi-fluid (lines) and the classical theory (diamonds) solutions for a planar plasma-sheath transition with a floating wall. The orange is used for the ion variables, the black for the electrons, and the blue for the potential. We present the solution for the densities (top left), potential (top right), velocity (bottom left), and flux (bottom rigth). For each panel we present $q=0.013$ (top), $q=0.0038$ (middle), and $q=0.0011$ (bottom). A zoom of the densities and the velocities in the sheath is also presented as a function of the distance to the wall in $\lambda_{s h}$. The position where $\bar{u}_{\mathfrak{i}}=\bar{u}_{B}^{\text {mod }}$ is marked in those plots as a green line. The simulations consider an argon gas with $m_{\mathfrak{e}} / m_{i}=1.37 \cdot 10^{-5}$ and $T_{\mathfrak{i}} / T_{\mathfrak{e}}=0.025$ 


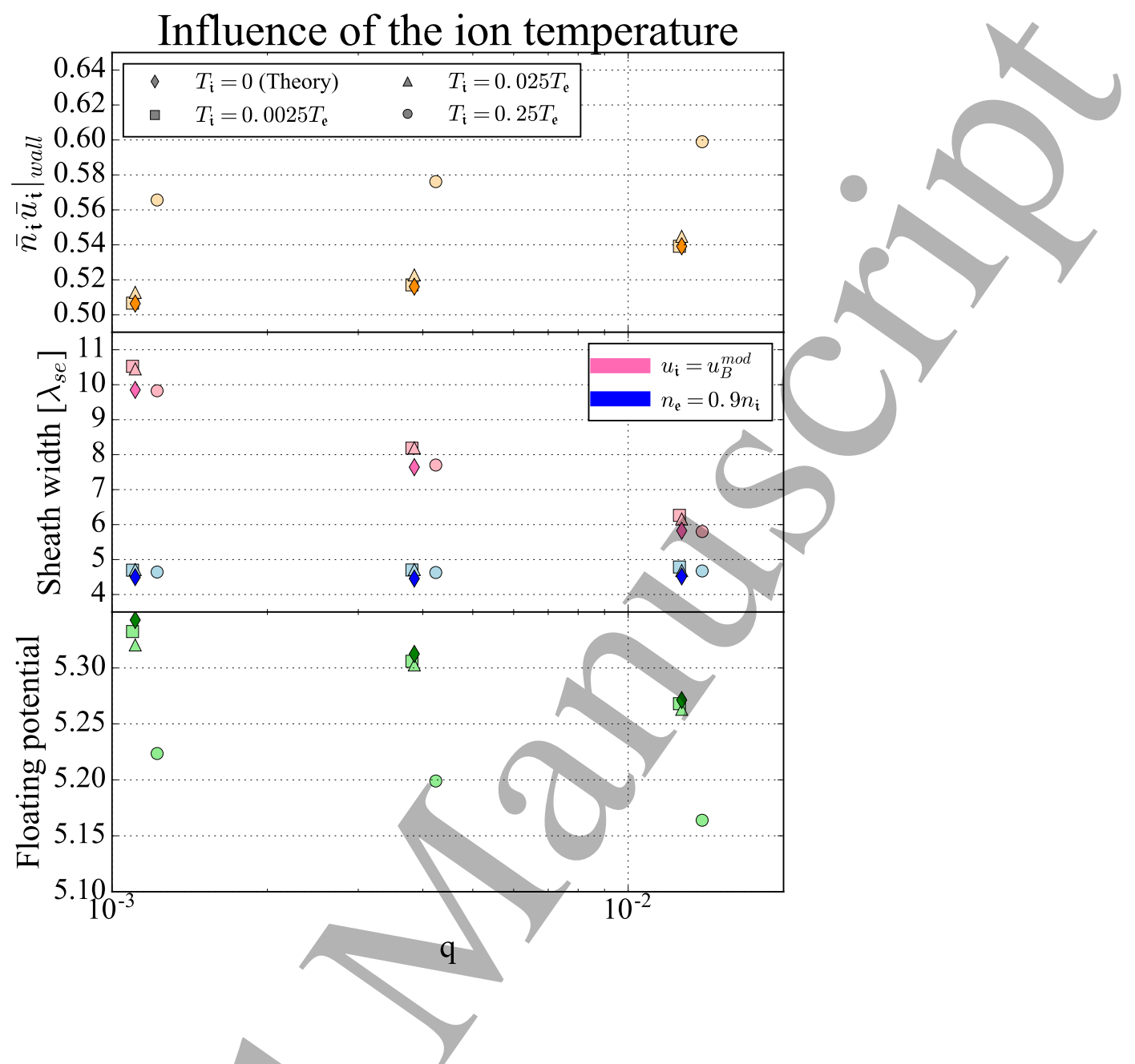

Figure 3. Ion wall flux (top), the sheath width (middle), and the value for the floating potential (bottom) as a function of the $q$-parameter in an argon plasma for three ion temperatures $\kappa=0.0025$ (squares), $\kappa=0.025$ (triangles), and $\kappa=0.25$ (circles). The multi-fluid solutions are compared with the classical theory (diamonds). The sheath width is computed with two criteria, the position where the ions cross Bohm's speed (pink) and where the electron density is 0.9 times the ion density (blue). 


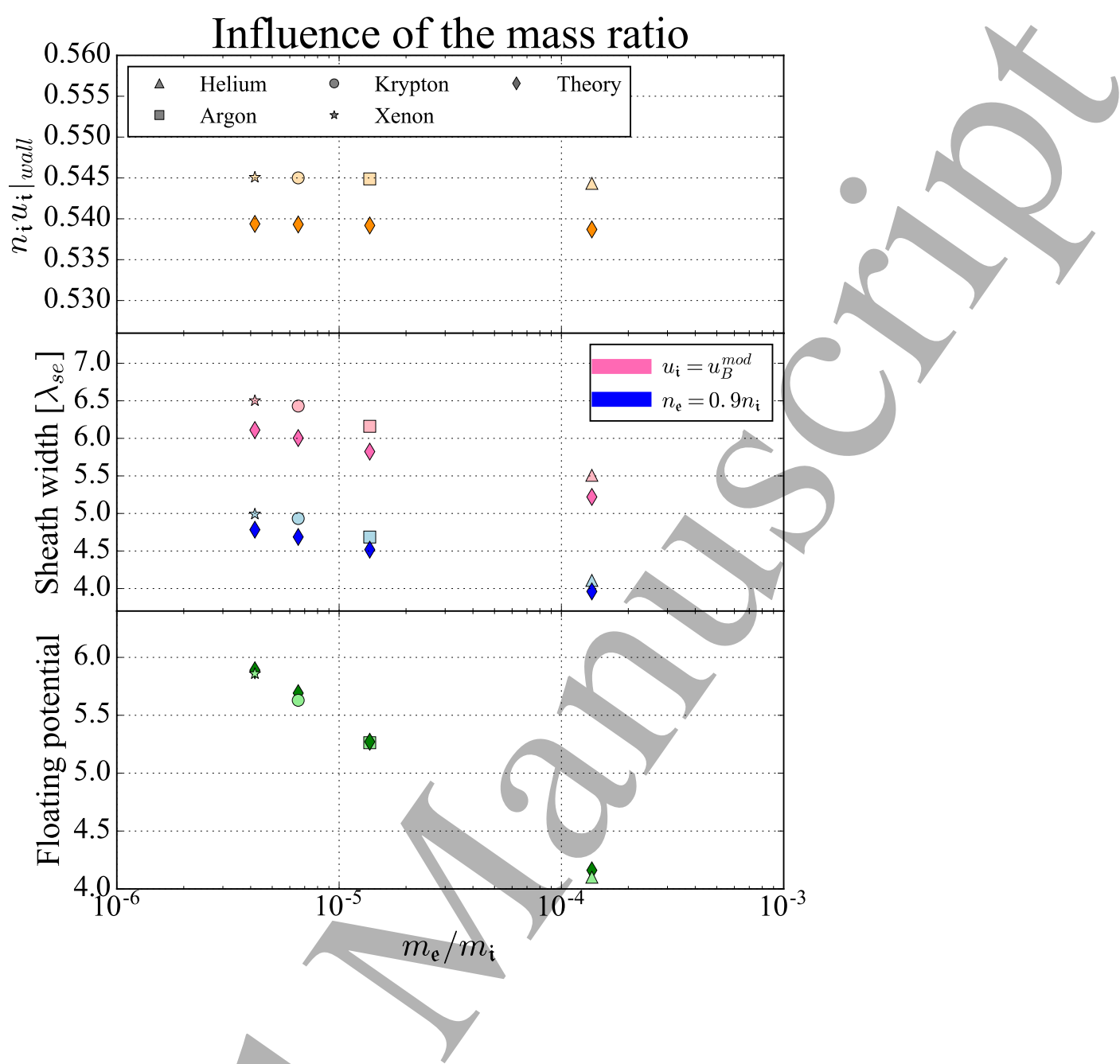

Figure 4. Ion wall flux (top), the sheath width (middle), and the value for the floating potential (bottom) as a function of the electron-to-ion mass ratio for four different ions: helium (triangles), argon (squares), krypton (circles), and xenon (stars). The multifluid solutions are compared with the classical theory (diamonds). The sheath width is computed with two criteria, the position where the ions cross Bohm's speed (pink) and where the electron density is 0.9 times the ion density (blue). 


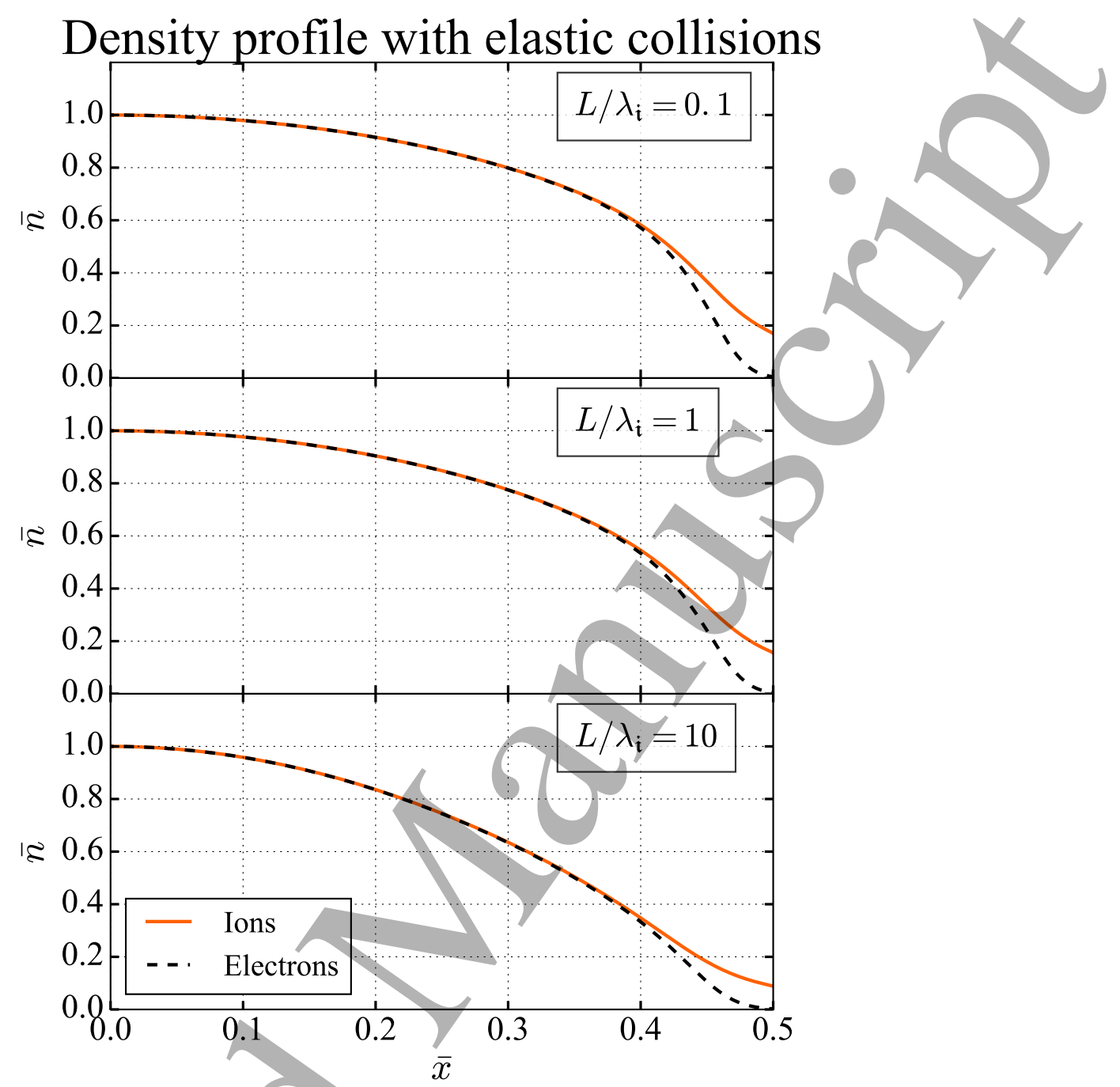

Figure 5. Density profile for different collisional levels $L / \lambda_{\mathfrak{i}}=0.1$ (top), $L / \lambda_{\mathfrak{i}}=1$ (middle), and $L / \lambda_{\mathfrak{i}}=10$ (lower) for an argon plasma with $T_{\mathfrak{i}}=0.025 T_{\mathfrak{e}}$ and $L=100 \lambda_{D 0}$. 


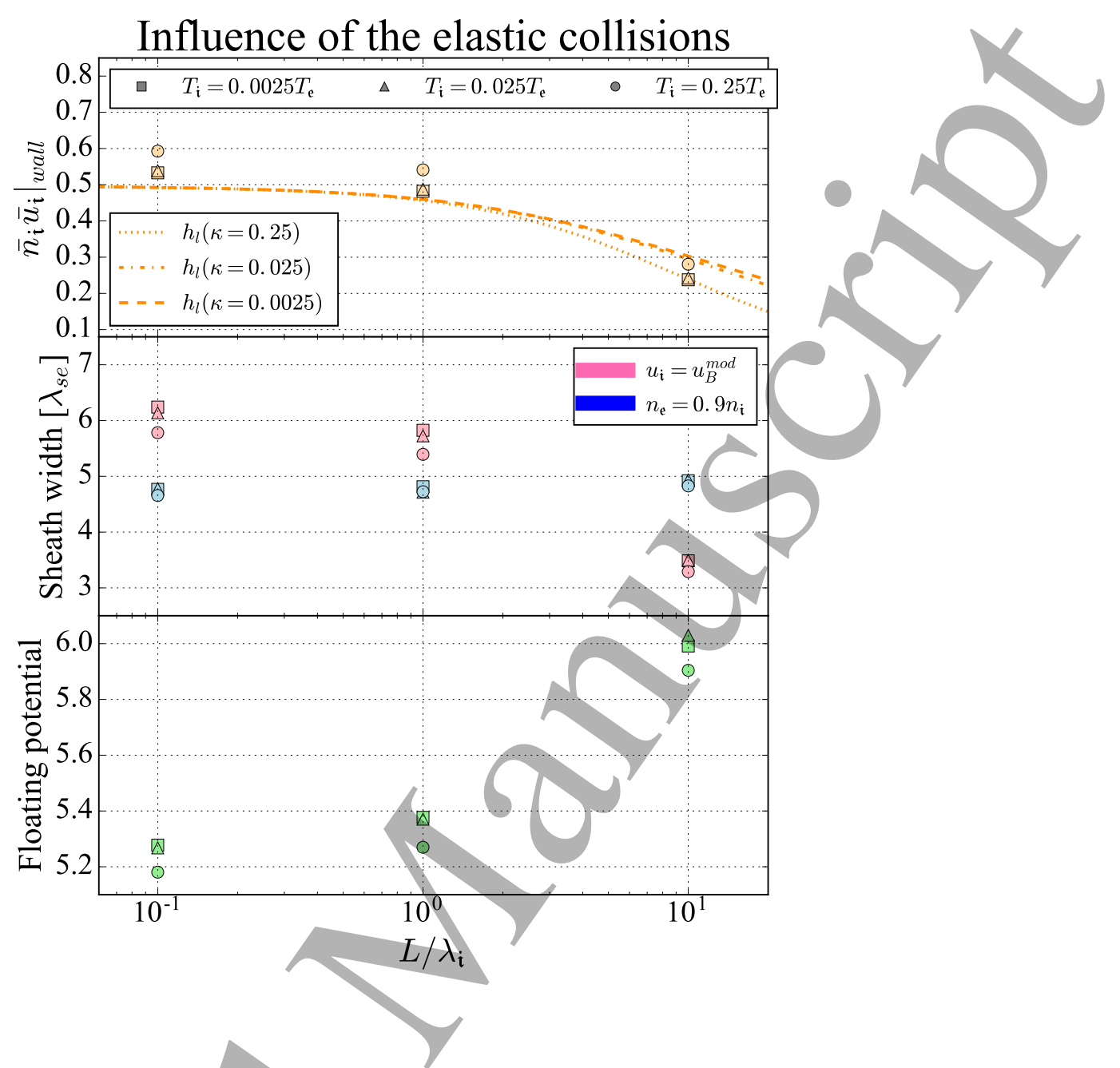

Figure 6. Ion wall flux (top), the sheath width (middle), and the value for the floating potential (bottom) as a function of the inverse of the normalized ion mean-free-path for three different temperatures: $\kappa=0.0025$ (squares), $\kappa=0.025$ (triangles), and $\kappa=0.25$ (circles). The lines correspond to the heuristic expression for $h_{l}$ from [43]. 

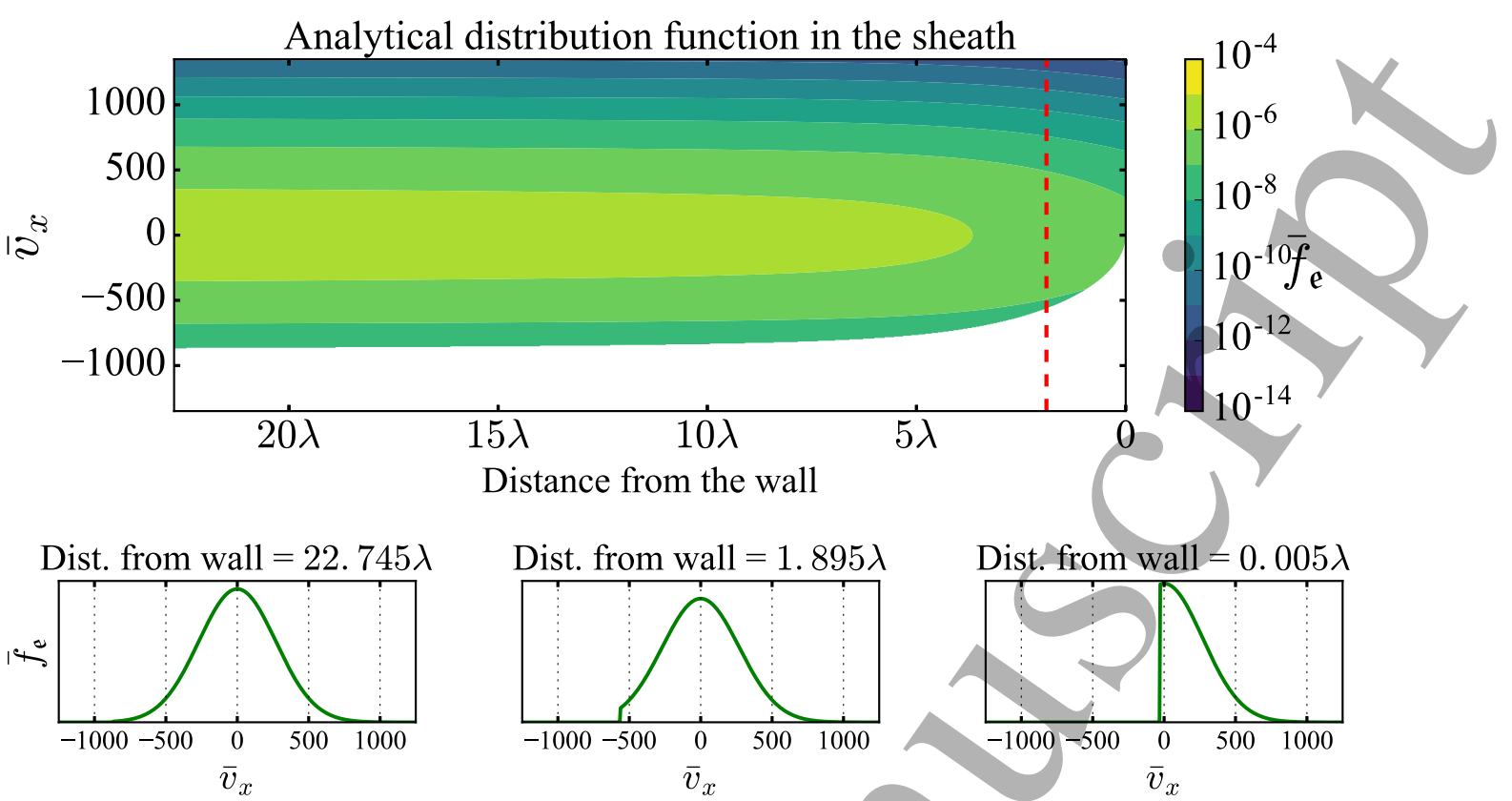

Figure 7. Analytical distribution function of a collisionless sheath with an absorbing wall. The distribution function is assumed to be Maxwellian at the edge of the sheath. 



Dist. from the wall
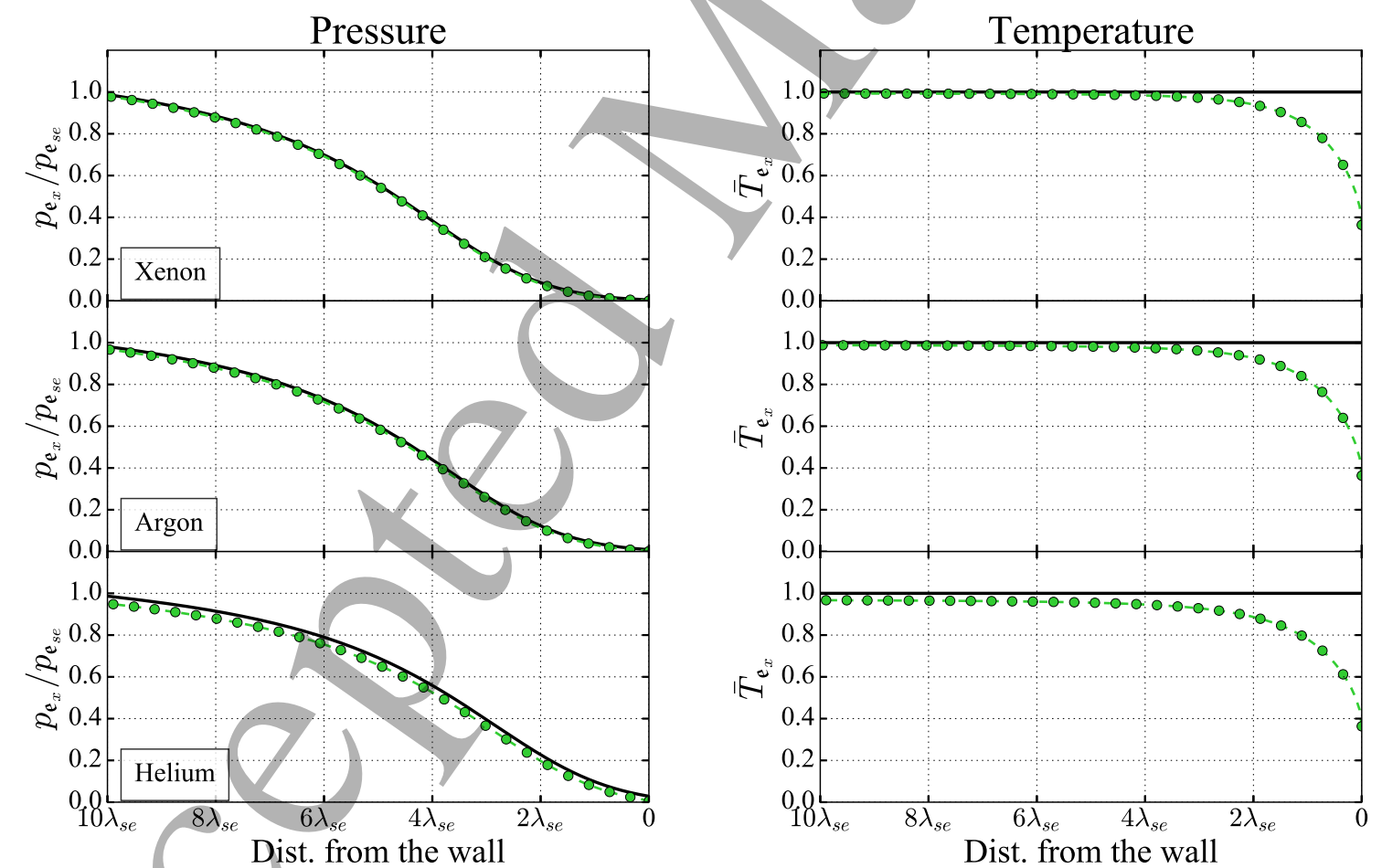

Figure 8. Comparison between the analytical moments of the truncated Maxwellian EVDF (circles) and the multi-fluid solution (solid line) for a xenon (top of every panel), argon (middle), and helium (bottom) plasma. The simulations use $\kappa=0.025$ and $L=1000 \lambda_{D 0}$. 